\title{
Approximating the chromatic index of multigraphs*
}

\author{
Guantao Chen† Xingxing Yuł Wenan Zang§
}

\begin{abstract}
It is well known that if $G$ is a multigraph then $\chi^{\prime}(G) \geq \chi^{\prime *}(G):=\max \{\Delta(G), \Gamma(G)\}$, where $\chi^{\prime}(G)$ is the chromatic index of $G, \chi^{\prime *}(G)$ is the fractional chromatic index of $G, \Delta(G)$ is the maximum degree of $G$, and $\Gamma(G)=\max \{2|E(G[U])| /(|U|-1): U \subseteq$ $V(G),|U| \geq 3,|U|$ is odd $\}$. The conjecture that $\chi^{\prime}(G) \leq \max \{\Delta(G)+1,\lceil\Gamma(G)\rceil\}$ was made independently by Goldberg (1973), Anderson (1977), and Seymour (1979). Using a probabilistic argument Kahn showed that for any $c>0$ there exists $D>0$ such that $\chi^{\prime}(G) \leq \chi^{\prime *}(G)+c \chi^{\prime *}(G)$ when $\chi^{\prime *}(G)>D$. Nishizeki and Kashiwagi proved this conjecture for multigraphs $G$ with $\chi^{\prime}(G)>\lfloor(11 \Delta(G)+8) / 10\rfloor$; and Scheide recently improved this bound to $\chi^{\prime}(G)>\lfloor(15 \Delta(G)+12) / 14\rfloor$. We prove this conjecture for multigraphs $G$ with $\chi^{\prime}(G)>\lfloor\Delta(G)+\sqrt{\Delta(G) / 2}\rfloor$, improving the above mentioned results. Our proof yields an algorithm for edge-coloring any multigraph $G$ using at most $\max \{\Delta(G)+\sqrt{\Delta(G) / 2},\lceil\Gamma(G)\rceil\}$ colors, which runs in polynomial time provided that $\Delta(G)$ is not part of the input. As a consequence, for multigraphs $G$ with $\chi^{\prime}(G)>\Delta(G)+\sqrt{\Delta(G) / 2}$ the answer to a 1964 problem of Vizing is affirmative.
\end{abstract}

\footnotetext{
*AMS Subject Classification: Primary 05C15, 05C85; Secondary 05C05, 05C70

${ }^{\dagger}$ Department of Mathematics and Statistics, Georgia State University, Atlanta, GA. This author is partially supported by NSF

${ }^{\ddagger}$ School of Mathematics, Georgia Institute of Technology, Atlanta, GA 30332. This author is partially supported by NSA and by NSFC Project 10628102

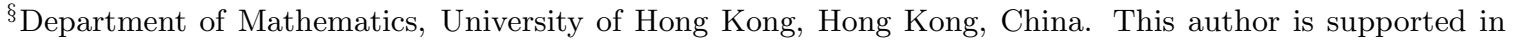
part by the Research Grants Council of Hong Kong
} 


\section{Introduction}

Let $G$ be a multigraph. A $k$-edge-coloring of $G$ is an assignment of $k$ colors to the edges of $G$ so that no two adjacent edges receive the same color. The chromatic index of $G$, denoted by $\chi^{\prime}(G)$, is the smallest $k$ for which $G$ admits a $k$-edge-coloring. Since it is $N P$-hard to determine $\chi^{\prime}(G)$ (see Holyer [9]), a good estimate of $\chi^{\prime}(G)$ has been the focus of extensive research.

Let $\Delta(G)$ denote the maximum degree of $G$. Clearly $\chi^{\prime}(G) \geq \Delta(G)$. A classical theorem of Shannon [22] asserts that $\chi^{\prime}(G) \leq 3 \Delta(G) / 2$. Vizing [26] and Gupta [7] proved that $\chi^{\prime}(G) \leq$ $\Delta(G)+\mu(G)$, where $\mu(G)$ is the maximum multiplicity of an edge of $G$; and Kierstead [12] studied the graphs $G$ for which $\chi^{\prime}(G)=\Delta(G)+\mu(G)$. This Vizing-Gupta result implies that if $G$ is a simple graph then $\chi^{\prime}(G) \in\{\Delta(G), \Delta(G)+1\}$.

Another lower bound for $\chi^{\prime}(G)$ is the fractional chromatic index defined below. Let

$$
\Gamma(G)=\max \left\{\frac{2|E(G[U])|}{|U|-1}: U \subseteq V(G),|U| \geq 3, \text { and }|U| \text { is odd }\right\}
$$

where $G[U]$ is the subgraph of $G$ induced by $U$. Observe that if $U \subseteq V(G)$ and $|U|$ is odd, then every matching in $G[U]$ has size at most $(|U|-1) / 2$. Consequently $\chi^{\prime}(G) \geq \Gamma(G)$; and hence $\chi^{\prime}(G) \geq \max \{\Delta(G), \Gamma(G)\}$. The number $\chi^{*}(G):=\max \{\Delta(G), \Gamma(G)\}$ is the fractional chromatic index of $G$ (see $[3,21]$ and $[23]$ ), which is the solution to a linear program. The fractional chromatic index can be determined in polynomial time by using the ellipsoid algorithm; since the corresponding separation problem is equivalent to the weighted matching problem, see Theorem 28.6 in [21].

In the 1970s, Goldberg [5], Anderson [1], and Seymour [23] independently (and in different forms) made the following conjecture.

Conjecture 1.1 For any multigraph $G, \chi^{\prime}(G) \leq \max \{\Delta(G)+1,\lceil\Gamma(G)\rceil\}$.

Note that Conjecture 1.1 if true implies that $\chi^{\prime}(G) \leq 1+\chi^{\prime *}(G)$ for all multigraphs $G$. When studying conjectures of Tutte and Fulkerson about factorizations of cubic graphs, Seymour [23] also made the following conjecture which is slightly weaker than Conjecture 1.1, but still achieves what Vizing's theorem does for simple graphs.

Conjecture 1.2 For any multigraph $G, \chi^{\prime}(G) \leq 1+\max \{\Delta(G),\lceil\Gamma(G)\rceil\}$.

Conjecture 1.2 has an equivalent formulation in terms of $r$-graphs. Let $r$ be a positive integer. A multigraph $G=(V, E)$ is called an $r$-graph if $G$ is $r$-regular and, for every $X \subseteq V$ with $|X|$ odd, the number of edges between $X$ and $V-X$ is at least $r$ (in particular, $|V|$ is even). Seymour [23] proved that Conjecture 1.2 is equivalent to the conjecture that if $G$ is an $r$-graph then $\chi^{\prime}(G) \leq r+1$.

In the past three decades, there has been extensive research on Conjecture 1.1, see for example $[2,4,6,8,11,13-20,24]$. (For more related work and references, we refer the reader to Kahn [11], Jenson and Toft [10], Schrijver [21], and Favrholdt, Stiebitz and Toft [4].) A number of results related to Conjecture 1.1 have been obtained. For example, Goldberg [5,6] showed $\chi^{\prime}(G) \leq \max \{\lfloor(9 \Delta(G)+6) / 8\rfloor,\lceil\Gamma(G)\rceil\}$. Nishizeki and Kashiwagi [16] proved $\chi^{\prime}(G) \leq$ $\max \left\{\left\lfloor\frac{11 \Delta(G)+8}{10}\right\rfloor,\lceil\Gamma(G)\rceil\right\}$; a shorter proof of this theorem can be found in Tashkinov [25]. This 
bound has recently been improved to $\max \{\lfloor(13 \Delta(G)+10) / 12\rfloor,\lceil\Gamma(G)\rceil\}$ by Favrholdt, Stiebitz and Toft [4], and further to $\max \{\lfloor(15 \Delta(G)+12) / 14\rfloor,\lceil\Gamma(G)\rceil\}$ by Scheide [20]. Conjecture 1.1 was proved by Seymour [24] for $K_{4}$-free graphs, by Marcotte [14, 15] for multigraphs with no $K_{5}^{-}$-minors, and by Plantholt and Tipnis [17] for multigraphs with sufficiently high maximum degree (relative to $|V(G)|$ and $\mu(G)$ ). Sanders and Steurer [19] showed that for any $\epsilon>0$ there is a polynomial time algorithm (dependent on $\epsilon$ ) for edge-coloring any multigraph $G$ using at most $(1+\epsilon) \chi^{\prime}(G)+O(1 / \epsilon)$ colors. Also, Plantholt [18] proved that $\chi^{\prime}(G) \leq$ $\chi^{\prime *}(G)+1+\sqrt{|V(G)| \log |V(G)| / 10}$ when $|V(G)|$ is sufficiently large, and pointed out that his proof does not give a polynomial time coloring algorithm. On the other hand, using a sophisticated probabilistic argument, Kahn [11] proved that for any $c>0$ there exists $D>0$ such that $\chi^{\prime}(G) \leq \chi^{\prime *}(G)+c \chi^{\prime *}(G)$ when $\chi^{\prime *}(G)>D$.

The purpose of this paper is to establish the following result.

Theorem 1.3 For any multigraph $G, \chi^{\prime}(G) \leq \max \{\Delta(G)+\sqrt{\Delta(G) / 2},\lceil\Gamma(G)\rceil\}$. Moreover, there is an $O\left(V^{O(\sqrt{\Delta})+3} \Delta E\right)$ algorithm for edge-coloring any multigraph $G$ using at most $\max \{\Delta(G)+\sqrt{\Delta(G) / 2},\lceil\Gamma(G)\rceil\}$ colors.

When $\Delta$ is not part of the input, such an algorithm runs in polynomial time.

Note that Theorem 1.3 holds trivially when $\Delta(G) \leq 2$. So throughout the rest of this paper, we may assume $\Delta(G) \geq 3$ when needed.

As an immediate consequence of Theorem 1.3, Conjecture 1.1 holds for multigraphs $G$ with $\chi^{\prime}(G)>\lfloor\Delta(G)+\sqrt{\Delta(G) / 2}\rfloor$. When $\Delta(G)$ is large, Theorem 1.3 improves the above mentioned result of Scheide. Since $\chi^{\prime *}(G) \geq \Delta(G), \chi^{\prime}(G) \leq \chi^{\prime *}(G)+\sqrt{\chi^{\prime *}(G) / 2}$. Hence, our result also implies the above mentioned results of Kahn and of Plantholdt (when $\Delta(G) \leq$ $|V(G)| \log |V(G)|)$

Let $G$ be a graph whose edges are properly colored. Following [10], an interchange with respect to (distinct) colors $\alpha$ and $\beta$ consists in swapping the colors on the edges in a component of the subgraph of $G$ induced by all edges with color $\alpha$ or $\beta$. In 1964, Vizing [26] (also see [10]) asked the following "interchange" problem.

Problem 1.4 Is it true that if $\chi^{\prime}(G) \geq \Delta(G)+2$ then given any edge-coloring of a multigraph $G$ one can obtain an optimal edge-coloring through a sequence of interchanges?

Our proof of Theorem 1.3 implies that the answer to Problem 1.4 is affirmative for graphs $G$ with $\chi^{\prime}(G) \geq \Delta(G)+\sqrt{\Delta(G) / 2}$.

Corollary 1.5 Let $G$ be a multigraph with $\chi^{\prime}(G) \geq \Delta(G)+\sqrt{\Delta(G) / 2}$. Then given any edgecoloring of $G$ one can obtain an optimal edge-coloring through a sequence of interchanges.

The proof technique we use is a generalization of Vizing's recoloring technique via interchanges. Recall Vizing's "fan sequence" (or "star") argument for proving that $\chi^{\prime}(G) \in$ $\{\Delta(G), \Delta(G)+1\}$ when $G$ is a simple graph. Take a partial edge-coloring of $G$ using $\Delta(G)+1$ colors (partial means that there may be uncolored edges), and pick an uncolored edge, say $a b_{1}$. From the edge $a b_{1}$, grow a fan (also known as star). Each time, we add a colored edge $a b_{i}(i \geq 2)$ whose color is missing at $b_{i-1}$ (i.e., not used by any edge incident with $\left.b_{i-1}\right)$. Since the graph is simple, this process must stop and give some "augmenting set" $\left\{a, b_{1}, \ldots, b_{k}\right\}$ 
(namely, two of its vertices have a common missing color). By applying an appropriate recoloring argument (a sequence of interchanges), we may produce a partial edge-coloring in which there is a color, say $\alpha$, missing at both $a$ and $b_{1}$, and we may then augment the set of colored edges by coloring $a b_{1}$ with $\alpha$. Continuing this process until all edges are colored, we obtain a $(\Delta(G)+1)$-edge-coloring of $G$. This recoloring scheme works because the graph is simple and because there are colors missing at every vertex.

When studying extremal graphs for the above mentioned Vizing-Gupta bound, Kierstead [12] used a similar process by growing a path instead of a star.

Tashkinov's approach in [25] for edge-coloring multigraphs generalizes the recoloring techniques of Vizing and Kierstead. Instead of growing a star or a path, a tree is grown from an uncolored edge $a b$; each time an edge is added to the tree, the color of that edge must be missing at some previous vertex in the tree. If the vertex set of the tree is "augmenting" then the edge-coloring can be modified through a complex recoloring procedure so that the edge $a b$ may be colored (without introducing a new color). If the growing process stops and the tree is not augmenting, then Tashkinov shows that the tree is small enough so that a case analysis can be carried out (but for this to work one needs at least $(11 \Delta(G)+8) / 10$ colors).

To prove Theorem 1.3, we need to grow a tree that is more complex than Tashkinov's, called VKT-trees (VKT stands for Vizing-Kierstead-Tashkinov). We start with Tashkinov's process. The key is to grow the tree when Tashkinov's process stops. In other words, when there is no choice we add to our tree an edge whose color is not missing at previous vertices of the tree. (Similar idea was also discussed in [4].) However, we need to pick such an edge carefully (called "connecting" edge). As we shall see, when we have at least $\Delta(G)+\sqrt{\Delta(G) / 2}$ colors, the number of connecting edges is less than $\sqrt{\Delta(G) / 2}$, and we can grow the tree in a way so that a recoloring argument can be used either to color more edges (without introducing a new color) or to show that the number of colors used so far is less than $\lceil\Gamma(G)\rceil$ (and hence we are free to introduce a new color).

The VKT-trees will be defined in section 2, where we also prove several simple properties about these trees. In particular, we show that if a VKT-tree cannot be grown further, then the number of colors used so far is less than $\lceil\Gamma(G)\rceil$. We also introduce two partial orderings on VKT-trees, to be used as measurements of VKT-trees after interchanges. In Section 3, we prove several recoloring lemmas using VKT-trees. We also show that when the number of colors is sufficiently large, one can choose colors satisfying certain properties (to be used to avoid certain colors during a recoloring process). In section 4 , we prove recoloring lemmas that transfer "bad" augmenting pairs to "good" ones, and use them to deal with VKT-trees containing augmenting pairs. In section 5, we show how to deal with VKT-trees with no augmenting pairs, and complete the proofs of Theorem 1.3 and Corollary 1.5.

\section{VKT-trees}

We begin with a few concepts and notation. Let $G$ be a graph. For $S \subseteq V(G)$, we use $G-S$ to denote the graph obtained from $G$ by deleting $S$ and the edges of $G$ incident with $S$, and we use $[S, G-S]$ to denote the set of edges of $G$ with exactly one end in $S$. For $S \subseteq E(G)$, $G-S$ is the graph obtained from $G$ by deleting $S$. If $S=\{s\}$, then we simply write $G-s$ instead of $G-\{s\}$. For $S \subseteq V(G) \cup E(G)$, we use $G[S]$ to denote the subgraph of $G$ with $V(G[S])=(S \cap V(G)) \cup\{u: u$ is incident with an edge in $S\}$ and $E(G[S])=(S \cap E(G)) \cup\{u v \in$ 
$E(G):\{u, v\} \subseteq S \cap V(G)\}$. When $S \subseteq V(G)$ or $S \subseteq E(G), G[S]$ is just the subgraph of $G$ induced by $S$ in the usual sense. If $S=\left\{x_{1}, \ldots, x_{p}\right\}$ then we also write $G\left[x_{1}, \ldots, x_{p}\right]$ instead of $G[S]$. For any subgraph $T$ of $G$, we write $G[T]$ instead of $G[V(T)], G-T$ instead of $G-V(T)$, and $[T, G-T]$ instead of $[V(T), G-V(T)]$. For $H \subseteq G$ and $S \subseteq E(G)$, we use $H+S$ to denote the subgraph of $G$ obtained from $H$ by adding $S$ and all incident vertices. When $S=\{s\}$, we simply write $H+s$.

Let $G$ be a graph and $\mathcal{C}$ a set of colors. A partial edge-coloring of $G$ using colors from $\mathcal{C}$ is a function $c: S \rightarrow \mathcal{C}$, where $S \subseteq E(G)$, such that for any $e, f \in S, c(e) \neq c(f)$ whenever $e$ and $f$ are adjacent in $G$. The set $S$ is usually denoted by $E_{G}(c)$, or $E(c)$ when $G$ is understood. If $|\mathcal{C}|=k$, then $c$ is called a partial $k$-edge-coloring of $G$. When $E(c)=E(G)$, then $c$ is just an edge-coloring of $G$ in the usual sense. Throughout this paper, the letter $c$ (with or without subscripts or superscripts) will be used to name partial edge-colorings; and lowercase Greek letters (with or without subscripts) will be used to denote colors.

Let $G$ be a graph and let $c$ be a partial edge-coloring of $G$, using colors from the collection of colors $\mathcal{C}$. For any distinct $\alpha, \beta \in \mathcal{C}$, we define $G_{c}(\alpha, \beta):=G[\{e \in E(c): c(e) \in\{\alpha, \beta\}\}]$; and write $G(\alpha, \beta):=G_{c}(\alpha, \beta)$ when $c$ is understood. The components of $G(\alpha, \beta)$ are paths or cycles. For any component $D$ of $G_{c}(\alpha, \beta)$, we say that the partial edge-coloring $c^{\prime}$ of $G$ is obtained from $c$ by an interchange on $D$ (or interchanging $D$ ) if $c^{\prime}(e)=c(e)$ for all $e \in E(c)-E(D), c^{\prime}(e)=\alpha$ for all $e \in E(D)$ with $c(e)=\beta$, and $c^{\prime}(e)=\beta$ for all $e \in E(D)$ with $c(e)=\alpha$. For any $x \in V(G)$, we use $M_{\mathcal{C}, c}(x)$ to denote the set of colors in $\mathcal{C}$ that are not used by any edge incident with $x$. Usually, $\mathcal{C}$ will be fixed; so we often write $M_{c}(x)$ instead of $M_{\mathcal{C}, c}(x)$, which stands for the set of colors missing at $x$. For a subgraph $H$ of $G$, we write $M_{c}(H):=\bigcup_{x \in V(H)} M_{c}(x)$ and $c(H)=\{c(e): e \in E(H)\}$.

Definition 2.1 Let $G$ be a graph, and let $c$ be a partial edge-coloring of $G$. The nonempty set $S \subseteq V(G)$ is said to be nonaugmenting with respect to $c$ if, for any distinct $x, y \in S$, $M_{c}(x) \cap M_{c}(y)=\emptyset$; and augmenting with respect to $c$ otherwise. (This concept was implicit in Vizing [26] and Goldberg [6].) We say that the set $S \subseteq V(G)$ is critical with respect to $c$ if

(i) for any color $\alpha \in M_{c}(S)$, no edge in $[S, G-S]$ uses the color $\alpha$, and

(ii) for any color $\alpha \notin M_{c}(S)$, at most one edge in $[S, G-S]$ uses the color $\alpha$.

Proposition 2.2 Let $G$ be a graph, let c be a partial $k$-edge-coloring of $G$, and let $S \subseteq V(G)$. Suppose $M_{c}(S) \neq \emptyset$ and $S$ is both nonaugmenting and critical (with respect to $\left.c\right)$. Then $\Gamma(G[S]) \geq k$.

Proof. Let $\alpha$ be an arbitrary color used by $c$. Suppose $\alpha \in M_{c}(S)$. Then, because $S$ is critical with respect to $c$, no edge in $[S, G-S]$ uses color $\alpha$. Since $S$ is nonaugmenting with respect to $c, \alpha \in M_{c}(x)$ for a unique $x \in S$. Therefore, $G[S]-x$ has a perfect matching whose edges are all colored with $\alpha$. In particular, $|S|$ is odd.

Now assume $\alpha \notin M_{c}(S)$. Then because $S$ is critical with respect to $c$ and $|S|$ is odd, exactly one edge in $[S, G-S]$ uses color $\alpha$. Let $u v$ be the unique edge in $[S, G-S]$ such that $u \in S$ and $c(u v)=\alpha$. Then $G[S]-u$ has a perfect matching whose edges are colored $\alpha$.

Therefore, we see that $E(G[S])$ contains a union of $k$ edge-disjoint matchings of size $(|S|-$ 1) $/ 2$. So $\Gamma(G[S]) \geq k$. 
We say that $(G, a b, c)$ is a triple if $G$ is a connected graph, $c$ is a partial edge-coloring of $G$, and $a b \in E(G)-E(c)$. If, in addition, $c$ is a partial $k$-edge-coloring, then $(G, a b, c)$ is said to be a $k$-triple. We now define the VKT-trees to be used to prove Theorem 1.3. Note that condition (ii) in this definition describes which edge can be added in order to grow our tree when Tashkinov's process stops. By a path from a vertex $u$ to an edge $e$, we mean a path from $u$ to an end of $e$ but not containing the other end of $e$.

Definition 2.3 Let $(G, a b, c)$ be a triple, and let $T$ be a tree in $G$ with edges $e_{1}, \ldots, e_{m}$. We say that $(T, c)$ is a VKT-tree in $(G, a b, c)$ with edge ordering $e_{1}, \ldots, e_{m}$ if

(i) $e_{1}=a b,\left\{e_{2}, \ldots, e_{m}\right\} \subseteq E(c)$, and, for each $1 \leq i \leq m, T_{i}:=T\left[e_{1}, \ldots, e_{i}\right]$ is a tree, and

(ii) if $c\left(e_{i}\right) \notin M_{c}\left(T_{i-1}\right)$ (for each $2 \leq i \leq m$ ), then

(a) $c(e) \notin M_{c}\left(T_{i-1}\right)$ for all $e \in E(c) \cap\left[T_{i-1}, G-T_{i-1}\right]$,

(b) at least two edges in $\left[T_{i-1}, G-T_{i-1}\right]$ use the color $c\left(e_{i}\right)$, and

(c) if $S_{0}(T, c)$ denotes the maximal sequence $\left(e_{1}, \ldots, e_{k}\right)$ such that $c\left(e_{j}\right) \in M_{c}\left(T_{j-1}\right)$ for $2 \leq j \leq k$, and if $V_{0}(T, c)$ denotes the set of vertices incident with edges in $S_{0}(T, c)$, then there exist $x_{i} \in V_{0}(T, c)$ and $\alpha_{i} \in M_{c}\left(x_{i}\right)-c\left(T_{i}\right)$ such that $G\left[T_{i}\right] \cap G_{c}\left(\alpha_{i}, c\left(e_{i}\right)\right)$ contains a path from $x_{i}$ to $e_{i}$.

If, in addition, $c(e) \notin M_{c}(T)$ for each $e \in E(c) \cap[T, G-T]$, then $(T, c)$ is said to be complete. Any edge $e_{i}$ with $c\left(e_{i}\right) \notin M_{c}\left(T_{i-1}\right)$ is said to be connecting in $(T, c)$. Note that whenever $c\left(e_{i}\right) \notin M_{c}\left(T_{i-1}\right), V_{0}(T, c) \subseteq V\left(T_{i-1}\right)$. A pair of distinct vertices, say $\{x, y\}$, in $(T, c)$ is said to be divided if there is a connecting edge $e_{i}$ such that $x, y$ belong to different components of $T-e_{i}$; and is undivided otherwise. For notational simplicity, we also write $T_{0}:=\emptyset$ and $c\left(e_{1}\right) \in M_{c}\left(T_{0}\right)$.

Note that because of (a) the path in $G\left[T_{i}\right] \cap G_{c}\left(\alpha_{i}, c\left(e_{i}\right)\right)$ is contained in $G\left[T_{i-1}\right]$. Before introducing further concepts related to VKT-trees, we make two simple observations, which should help the reader digest the concept of VKT-trees. The first observation says that any "rooted" subtree of a VKT-tree is also a VKT-tree.

Lemma 2.4 Let $(G, a b, c)$ be a triple, and let $(T, c)$ be a VKT-tree in $(G, a b, c)$ with edge ordering $e_{1}, \ldots, e_{m}$. Then, for each $1 \leq s \leq m,\left(T_{s}, c\right)$ is also a VKT-tree in $(G, a b, c)$, and any edge of $T_{s}$ is connecting in $\left(T_{s}, c\right)$ iff it is connecting in $(T, c)$.

Proof. Clearly, $T_{1}$ (with only one edge $e_{1}$ ) satisfies Definition 2.3(i), and Definition 2.3(ii) does not apply to $T_{1}$. So $\left(T_{1}, c\right)$ is a VKT-tree in $(G, a b, c)$. Now assume $s \geq 2$. Note that for each $1 \leq i \leq s, T_{i}$ is a subtree of $T$. Since $e_{1} \notin E(c)$ and $\left\{e_{2}, \ldots, e_{s}\right\} \subseteq E(c),\left(T_{s}, c\right)$ satisfies Definition 2.3(i).

To show that Definition 2.3(ii) holds for $\left(T_{s}, c\right)$, we pick an arbitrary edge $e_{i}, 2 \leq i \leq s$, and assume $c\left(e_{i}\right) \notin M_{c}\left(T_{i-1}\right)$. Then $S_{0}\left(T_{s}, c\right)=S_{0}(T, c)$ and $V_{0}\left(T_{s}, c\right)=V_{0}(T, c)$. Since $(T, c)$ is a VKT-tree in $(G, a b, c)$, it follows from Definition 2.3(ii) that (a) for any $e \in E(c) \cap\left[T_{i-1}, G-\right.$ $\left.T_{i-1}\right]$, we have $c(e) \notin M_{c}\left(T_{i-1}\right)$, (b) at least two edges in $\left[T_{i-1}, G-T_{i-1}\right]$ use the color $c\left(e_{i}\right)$, and (c) there exist $x_{i} \in V_{0}(T, c)=V_{0}\left(T_{s}, c\right)$ and $\alpha_{i} \in M_{c}\left(x_{i}\right)-c\left(T_{i}\right)$ such that $G\left[T_{i}\right] \cap G_{c}\left(\alpha_{i}, c\left(e_{i}\right)\right)$ contains a path from $x_{i}$ to $e_{i}$. 
So $\left(T_{s}, c\right)$ is also a VKT-tree in $(G, a b, c)$. Clearly, any edge of $T_{s}$ is connecting in $\left(T_{s}, c\right)$ iff it is connecting in $(T, c)$.

The next observation shows when a VKT-tree may be extended by adding an edge.

Lemma 2.5 Let $(G, a b, c)$ be a triple, let $(T, c)$ be a VKT-tree in $(G, a b, c)$ with edge ordering $e_{1}, \ldots, e_{m}$, and let $e \in E(c) \cap[T, G-T]$. Suppose either

(a) $c(e) \in M_{c}(T)$, or

(b) $c(e) \notin M_{c}(T),(T, c)$ is complete, at least two edges in $[T, G-T]$ use the color $c(e)$, and there exist $x \in V_{0}(T, c)$ and $\alpha \in M_{c}(x)-c(T+e)$ such that $G[T+e] \cap G_{c}(\alpha, c(e))$ contains a path from $x$ to $e$.

Then $(T+e, c)$ is a VKT-tree in $(G, a b, c)$ with edge ordering $e_{1}, \ldots, e_{m}, e$, and any edge of $T$ is connecting in $(T, c)$ iff it is connecting in $(T+e, c)$.

Proof. Because $e \in E(c) \cap[T, G-T], T+e$ with edge ordering $e_{1}, \ldots, e_{m}, e$ satisfies Definition 2.3(i). To prove that $(T+e, c)$ also satisfies Definition 2.3(ii), choose an arbitrary edge $e_{i}$ with $1 \leq i \leq m+1$ where $e_{m+1}=e$, and assume $c\left(e_{i}\right) \notin M_{c}\left(T_{i-1}\right)$.

Since $(T, c)$ is a VKT-tree in $(G, a b, c)$, the following holds when $i \leq m$ : For any $f \in$ $E(c) \cap\left[T_{i-1}, G-T_{i-1}\right]$ we have $c(f) \notin M_{c}\left(T_{i-1}\right)$; at least two edges in $\left[T_{i-1}, G-T_{i-1}\right]$ use the color $c\left(e_{i}\right)$; and there exist $x_{i} \in V_{0}(T, c)$ and $\alpha_{i} \in M_{c}\left(x_{i}\right)-c\left(T_{i}\right)$ such that $G\left[T_{i}\right] \cap G_{c}\left(\alpha_{i}, c\left(e_{i}\right)\right)$ contains a path from $x_{i}$ to $e_{i}$. So Definition 2.3(ii) holds when $i \leq m$.

If (a) occurs then Definition 2.3(ii) does not apply to $e_{m+1}$; and in this case, $(T+e, c)$ is a VKT-tree in which $e$ is not connecting. If (b) occurs then $S_{0}(T+e, c)=S_{0}(T, c)$ and $V_{0}(T+e, c)=V_{0}(T, c)$, and Definition 2.3(ii) holds for $e_{m+1}$. Therefore, $(T+e, c)$ is a VKT-tree in $(G, a b, c)$ with edge ordering $e_{1}, \ldots, e_{m}, e$. Clearly, any edge of $T$ is connecting in $(T, c)$ iff it is connecting in $(T+e, c)$.

Note that if (a) occurs then $e$ is not connecting in $(T+e, c)$; and if (b) occurs then $e$ is connecting in $(T+e, c)$. We now extend the concept of nonaugmenting set to VKT-trees.

Definition 2.6 Let $(G, a b, c)$ be a triple, and let $(T, c)$ be a VKT-tree in $(G, a b, c)$. We say that $(T, c)$ is augmenting if there exist two distinct vertices $x, y$ of $T$ forming an augmenting pair in $(T, c)$, i.e., $M_{c}(x) \cap M_{c}(y) \neq \emptyset$. The augmenting pair $\{x, y\}$ is said to be good if there exists $\alpha \in M_{c}(x) \cap M_{c}(y)$ such that $\alpha$ is not used by any connecting edge in $(T, c)$. We say that $(T, c)$ is nonaugmenting if $(T, c)$ is not augmenting.

The following observation is a direct consequence of Definition 2.6 and Lemma 2.4.

Lemma 2.7 Let $(G, a b, c)$ be a triple and let $(T, c)$ be a nonaugmenting VKT-tree in $(G, a b, c)$ with edge ordering $e_{1}, \ldots, e_{m}$. Then for any $1 \leq s \leq m,\left(T_{s}, c\right)$ is a nonaugmenting VKT-tree in $(G, a b, c)$.

We shall see in section 5 that the existence of an augmenting pair in $(T, c)$ will enable us to augment the set of colored edges (without introducing a new color) through a sequence of interchanges, or to augment $(T, c)$ in a certain way.

Next we extend the concept of critical set to VKT-trees. 
Definition 2.8 Let $(G, a b, c)$ be a triple and let $(T, c)$ be a VKT-tree in $(G, a b, c)$. We say that $(T, c)$ is critical if

(i) $(T, c)$ is complete, and

(ii) for any $\alpha \notin M_{c}(T)$, precisely one edge in $E(c) \cap[T, G-T]$ uses the color $\alpha$.

The next lemma describes a situation where a new color should be introduced.

Lemma 2.9 Let $(G, a b, c)$ be a k-triple and let $(T, c)$ be a VKT-tree in $(G, a b, c)$. Suppose $(T, c)$ is both nonaugmenting and critical. Then $k<\Gamma(G[T]) \leq \chi^{\prime}(G)$.

Proof. By definition, $\Gamma(G[T]) \leq \Gamma(G) \leq \chi^{\prime}(G)$. Note that $V(T)$ is both nonaugmenting and critical. So by Proposition 2.2 and since $a b$ is not colored, $\Gamma(G[T]-a b) \geq k$. Hence, $\Gamma(G[T])>k$.

We now introduce further concepts about VKT-trees, which are needed to define two partial orderings of VKT-trees.

Definition 2.10 Let $(G, a b, c)$ be a triple and let $(T, c)$ be a VKT-tree in $G$ with edge ordering $e_{1}, \ldots, e_{m}$. The vertex incident with $e_{m}$ but not in $T_{m-1}$ is called the top of $(T, c)$. Let $0 \leq r \leq m$ with $r$ minimum such that $T\left[e_{r+1}, \ldots, e_{m}\right]$ is a path. Define $C(T, c):=T_{r}$ (the center of $(T, c)$ ), and $B(T, c):=T\left[e_{r+1}, \ldots, e_{m}\right]$ (the branch of $\left.(T, c)\right)$. The VKT-tree $(T, c)$ can be divided into segments according to its connecting edges:

- If there is no connecting edge in $(T, c)$ then $S_{0}(T, c)=\left(e_{1}, \ldots, e_{m}\right)$ and let $S(T, c)=\emptyset$;

- otherwise let $e_{i_{1}}, \ldots, e_{i_{n}}$ be the connecting edges in $(T, c)\left(2 \leq i_{1}<\ldots<i_{n} \leq m\right)$, then $S_{0}(T, c)=\left(e_{1}, \ldots, e_{i_{1}-1}\right)$; and we define

$$
\begin{aligned}
& -S(T, c):=\left(e_{1}, e_{2}, \ldots, e_{i_{n}}\right), \\
& -S_{j}(T, c):=\left(e_{i_{j}}, \ldots, e_{i_{j+1}-1}\right) \text { for } 1 \leq j \leq n-1, \text { and } \\
& -S_{n}(T, c):=\left(e_{i_{n}}, \ldots, e_{m}\right) .
\end{aligned}
$$

For $0 \leq j \leq n, S_{j}(T, c)$ is called the $j$ th segment of $(T, c) . S_{n}(T, c)$ is also called the last segment of $(T, c)$, and $e_{i_{n}}$ is called the last connecting edge in $(T, c)$.

We conclude this section by defining two partial orders $<_{1}$ and $<_{2}$ on VKT-trees in triples. These partial orders will be used to measure whether we have "reduced" or "augmented" a given VKT-tree $(T, c)$ to a new VKT-tree $\left(T^{\prime}, c^{\prime}\right)$. The sequence $\left(a_{1}, \ldots, a_{m}\right)$ is a truncation of the sequence $\left(b_{1}, \ldots, b_{n}\right)$ if $m \leq n$ and $a_{i}=b_{i}$ for all $1 \leq i \leq m$; and if $m<n$ then $\left(a_{1}, \ldots, a_{m}\right)$ is said to be a proper truncation of $\left(b_{1}, \ldots, b_{n}\right)$.

Definition 2.11 Let $(G, a b, c)$ and $\left(G, a b, c^{\prime}\right)$ be two triples such that $E(c)=E\left(c^{\prime}\right)$, and $c$ and $c^{\prime}$ use the same set of colors. Let $(T, c)$ and $\left(T^{\prime}, c^{\prime}\right)$ be VKT-trees in $(G, a b, c)$ and $\left(G, a b, c^{\prime}\right)$, respectively.

- We write $\left(T^{\prime}, c^{\prime}\right)<_{1}(T, c)$ if either 
(i) $C\left(T^{\prime}, c^{\prime}\right)$ is a proper subgraph of $C(T, c)$, or

(ii) $C(T, c)=C\left(T^{\prime}, c^{\prime}\right)$ and $B\left(T^{\prime}, c^{\prime}\right)$ is a proper subgraph of $B(T, c)$.

- We write $(T, c)<_{2}\left(T^{\prime}, c^{\prime}\right)$ if there exists an integer $p \geq 0$ such that

(i) $S_{p}(T, c)$ is not the last segment of $(T, c)$,

(ii) $S_{j}(T, c)=S_{j}\left(T^{\prime}, c^{\prime}\right)$ for $0 \leq j \leq p-1$, and

(iii) $S_{p}(T, c)$ is a proper truncation of $S_{p}\left(T^{\prime}, c^{\prime}\right)$.

Note that when $(T, c)<_{2}\left(T^{\prime}, c^{\prime}\right),(T, c)$ must have a connecting edge (since $S_{p}(T, c)$ is not the last segment of $(T, c))$; and for any edge in $\bigcup_{j=0}^{p} S_{j}\left(T^{\prime}, c^{\prime}\right)$, it is connecting in $\left(T^{\prime}, c^{\prime}\right)$ iff it is connecting in $(T, c)$. It is easy to verify that $<_{1}$ and $<_{2}$ induce partial orderings on VKT-trees in triples. The partial order $<_{1}$ is implicitly used in [25]. Note that when $\left(T^{\prime}, c^{\prime}\right)<_{1}(T, c), T^{\prime}$ need not be a subtree of $T$, but $C\left(T^{\prime}, c^{\prime}\right)$ is always a truncation of $(T, c)$.

\section{Interchange lemmas}

In this section we prove several lemmas concerning the effect on VKT-trees when interchanges are performed. Our first interchange lemma is a simple observation, which describes a situation where an interchange preserves connecting edges.

Lemma 3.1 Let $(G, a b, c)$ be a triple, and let $(T, c)$ be a VKT-tree in $(G, a b, c)$ with edge ordering $e_{1}, \ldots, e_{m}$. Let $\alpha, \beta$ be distinct colors used by $c$, let $A$ be a component of $G_{c}(\alpha, \beta)$ such that $A \subseteq G-T_{m-1}$, and let $c^{\prime}$ be the partial edge-coloring of $G$ obtained from $c$ by interchanging $A$. Then

(1) $\left(T, c^{\prime}\right)$ is a VKT-tree in $\left(G, a b, c^{\prime}\right)$ with edge ordering $e_{1}, \ldots, e_{m}$, and

(2) any edge of $T$ is connecting in $\left(T, c^{\prime}\right)$ iff it is connecting in $(T, c)$.

Proof. Clearly, $\left(T, c^{\prime}\right)=(T, c)$, with edge ordering $e_{1}, \ldots, e_{m}$, satisfies Definition 2.3(i). Since $A \subseteq G-T_{m-1}, M_{c^{\prime}}(x)=M_{c}(x)$ for all $x \in V\left(T_{m-1}\right)$ and $c^{\prime}(e)=c(e)$ for all $e \notin G-T_{m-1}$. Thus, Definition 2.3(ii)(a) and Definition 2.3(ii)(b) hold whenever $c^{\prime}\left(e_{i}\right) \notin M_{c^{\prime}}\left(T_{i-1}\right)$. Moreover, for $1 \leq i \leq m, c^{\prime}\left(e_{i}\right) \in M_{c^{\prime}}\left(T_{i-1}\right)$ iff $c\left(e_{i}\right) \in M_{c}\left(T_{i-1}\right)$. So (2) holds, $S_{0}(T, c)=S_{0}\left(T, c^{\prime}\right)$, and $V_{0}(T, c)=V_{0}\left(T, c^{\prime}\right)$.

To show that $\left(T, c^{\prime}\right)$ also satisfies Definition 2.3(ii)(c), assume $c^{\prime}\left(e_{i}\right) \notin M_{c^{\prime}}\left(T_{i-1}\right)$. Then $c\left(e_{i}\right) \notin M_{c}\left(T_{i-1}\right)$. Since $(T, c)$ is a VKT-tree, there exist $x_{i} \in V_{0}\left(T, c^{\prime}\right)=V_{0}(T, c)$ and $\alpha_{i} \in$ $M_{c^{\prime}}\left(x_{i}\right)-c^{\prime}\left(T_{i}\right)=M_{c}\left(x_{i}\right)-c\left(T_{i}\right)$ such that $G\left[T_{i}\right] \cap G_{c^{\prime}}\left(\alpha_{i}, c^{\prime}\left(e_{i}\right)\right)=G\left[T_{i}\right] \cap G_{c}\left(\alpha_{i}, c\left(e_{i}\right)\right)$ contains a path from $x_{i}$ to $e_{i}$. So Definition 2.3(ii)(c) holds for $\left(T, c^{\prime}\right)$. Hence $\left(T, c^{\prime}\right)$ is a VKT-tree in $\left(G, a b, c^{\prime}\right)$, and (1) holds.

It is clear that it takes $O(V)$ time to get $c^{\prime}$ from $c$ (when $A$ is given). The following lemma will enable us to avoid certain parts of a VKT-tree when performing interchanges.

Lemma 3.2 Let $(G, a b, c)$ be a triple, and let $(T, c)$ be a VKT-tree in $(G, a b, c)$ with edge ordering $e_{1}, \ldots, e_{m}$. Suppose $x \in V_{0}(T, c)$ and $\alpha \in M_{c}(x)-c(T)$. Then one of the following holds. 
(1) For each connecting edge $e_{s}$ in $(T, c)$ and for each color $\beta \notin M_{c}\left(T_{s-1}\right)$, the component of $G_{c}(\alpha, \beta)$ containing $x$ contains all edges in $\left[T_{s-1}, G-T_{s-1}\right]$ with color $\beta$.

(2) There exists a partial $k$-edge-coloring $c^{\prime}$ of $G$ obtained from $c$ by an interchange and there is a VKT-tree $\left(T^{\prime}, c^{\prime}\right)$ in $\left(G, a b, c^{\prime}\right)$ such that $(T, c)<_{2}\left(T^{\prime}, c^{\prime}\right)$.

Proof. Suppose (1) fails. Then there exist some connecting edge $e_{s}$ in $(T, c)$, a color $\beta \notin$ $M_{c}\left(T_{s-1}\right)$, and an edge $e \in\left[T_{s-1}, G-T_{s-1}\right]$ such that $c(e)=\beta$, and $e$ is not contained in the component of $G_{c}(\alpha, \beta)$ containing $x$. We choose such $e_{s}$ that $s$ is minimum. Thus, if $1 \leq i<s$ and $e_{i}$ is a connecting edge in $(T, c)$, then for any $\gamma \notin M_{c}\left(T_{i-1}\right)$ and for any $f \in\left[T_{i-1}, G-T_{i-1}\right]$ with $c(f)=\gamma, f$ is contained in the component of $G_{c}(\alpha, \gamma)$ containing $x$.

Let $D$ denote the component of $G_{c}(\alpha, \beta)$ containing $e$, and let $c^{\prime}$ denote the partial $k$-edgecoloring of $G$ obtained from $c$ by interchanging $D$.

Suppose $e_{s}$ is the first connecting edge in $(T, c)$. Then since $\beta \notin M_{c}\left(T_{s-1}\right), \beta \notin c\left(T_{s-1}\right)$. So $\{\alpha, \beta\} \cap c\left(T_{s-1}\right)=\emptyset$. Hence for $1 \leq i \leq s-1, c^{\prime}\left(e_{i}\right)=c\left(e_{i}\right) \in M_{c}\left(T_{i-1}\right)-\{\alpha, \beta\}=$ $M_{c^{\prime}}\left(T_{i-1}\right)-\{\alpha, \beta\}$. So by repeatedly applying Lemma $2.5(a),\left(T_{s-1}, c^{\prime}\right)$ is a VKT-tree in $\left(G, a b, c^{\prime}\right)$ (with no connecting edge). Since $c^{\prime}(e)=\alpha \in M_{c}(x)=M_{c^{\prime}}(x) \subseteq M_{c^{\prime}}\left(T_{s-1}\right)$, it follows from Lemma 2.5(a) that $\left(T_{s-1}+e, c^{\prime}\right)$ is a VKT-tree in $\left(G, a b, c^{\prime}\right)$ (with no connecting edge), and (2) holds with $T^{\prime}:=T_{s-1}+e$.

Thus we may assume that $e_{t}$ is the connecting edge in $(T, c)$ immediately preceding $e_{s}$. Then by the minimality of $s$ and since $\alpha \in M_{c}\left(V_{0}(T, c)\right), D \subseteq G-T_{t-1}$. So by Lemma 3.1, $\left(T_{t}, c^{\prime}\right)$ is a VKT-tree in $\left(G, a b, c^{\prime}\right)$, and any edge of $T_{t-1}$ is connecting in $\left(T_{t-1}, c^{\prime}\right)$ iff it is connecting in $(T, c)$. Since $e_{t}$ and $e_{s}$ are consecutive connecting edges in $(T, c), c\left(e_{j}\right) \in M_{c}\left(T_{j-1}\right)$ for $t+1 \leq j \leq s-1$. Since $\beta \notin M_{c}\left(T_{s-1}\right)$ and $\alpha \notin c(T),\{\alpha, \beta\} \cap\left\{c\left(e_{j}\right): t+1 \leq j \leq s-1\right\}=\emptyset$. Then $c^{\prime}\left(e_{j}\right)=c\left(e_{j}\right) \in M_{c}\left(T_{j-1}\right)-\{\alpha, \beta\}=M_{c^{\prime}}\left(T_{j-1}\right)-\{\alpha, \beta\}$ for $t+1 \leq j \leq s-1$. So by repeatedly applying Lemma $2.5(a)$, we see that $\left(T_{s-1}+e, c^{\prime}\right)$ is a VKT-tree in $\left(G, a b, c^{\prime}\right)$; and (2) holds with $T^{\prime}:=T_{s-1}+e$.

From the above proof and since $k \leq O(\Delta(G))$ (by definition, $\Gamma(G)=O(\Delta(G))$ ), we see that it takes $O(V \Delta)$ time to certify that Lemma 3.2(1) holds or find the $\left(T^{\prime}, c^{\prime}\right)$ in Lemma 3.2(2).

The next lemma says that if the last edge of an augmenting VKT-tree is connecting, then this VKT-tree can be augmented through $<_{2}$.

Lemma 3.3 Let $(G, a b, c)$ be a k-triple with $k \geq \Delta(G)+\sqrt{\Delta(G) / 2}$, let $(T, c)$ be a VKTtree in $(G, a b, c)$ with edge ordering $e_{1}, \ldots, e_{m}$, let $y$ be the top of $(T, c)$, and assume that $m \geq 2,\left(T_{m-1}, c\right)$ is nonaugmenting, and $y$ is in an augmenting pair in $(T, c)$. Then one of the following holds.

(1) $e_{m}$ is not a connecting edge in $(T, c)$.

(2) There is a partial $k$-edge-coloring $c^{\prime}$ of $G$ obtained from c by a sequence of at most two interchanges and there is a VKT-tree $\left(T^{\prime}, c^{\prime}\right)$ in $\left(G, a b, c^{\prime}\right)$ such that $(T, c)<_{2}\left(T^{\prime}, c^{\prime}\right)$.

Proof. Suppose $e_{m}$ is a connecting edge in $(T, c)$. Let $x \in V_{0}(T, c)$ and $\alpha_{m} \in M_{c}(x)-c(T)$ such that $G[T] \cap G_{c}\left(\alpha_{m}, c\left(e_{m}\right)\right)$ contains a path, say $P_{m}$, from $x$ to $e_{m}$.

We may assume $\alpha_{m} \in M_{c}(y)$. For otherwise, let $\alpha \in M_{c}(y) \cap M_{c}\left(T_{m-1}\right)-\left\{\alpha_{m}\right\}$ (since $\left(T_{m-1}, c\right)$ is nonaugmenting and $y$ is contained in an augmenting pair in $\left.(T, c)\right)$. Since $e_{m}$ is 
connecting in $(T, c)$, no edge in $\left[T_{m-1}, G-T_{m-1}\right]$ uses the colors $\alpha$ or $\alpha_{m}$. Thus the component $A$ of $G_{c}\left(\alpha, \alpha_{m}\right)$ containing $y$ is contained in $G-T_{m-1}$. Let $c^{\prime}$ denote the partial edge-coloring of $G$ obtained from $c$ by interchanging $A$. By Lemma 3.1, $\left(T, c^{\prime}\right)$ is a VKT-tree in $\left(G, a b, c^{\prime}\right)$ with edge ordering $e_{1}, \ldots, e_{m}$, and $\alpha_{m} \in M_{c^{\prime}}(y) \cap M_{c^{\prime}}(x)$. Since $A \subseteq G-T_{m-1}, P_{m}$ is a path in $G[T] \cap G_{c^{\prime}}\left(\alpha_{m}, c^{\prime}\left(e_{m}\right)\right)$ from $x$ to $e_{m}$, and we may simply use $c^{\prime}$ instead of $c$.

Therefore, since $\alpha_{m} \in M_{c}(y), P_{m}$ is a component of $G_{c}\left(\alpha_{m}, c\left(e_{m}\right)\right)$. So there is a component of $G_{c}\left(\alpha_{m}, c\left(e_{m}\right)\right)$, say $D$, intersecting $\left[T_{m-1}, G-T_{m-1}\right]$ and disjoint from $P_{m}$. We may assume that whenever $e_{i}$ is a connecting edge in $(T, c)$ and $i<m, P_{m}$ contains all edges in $\left[T_{i-1}, G-\right.$ $\left.T_{i-1}\right]$ with the color $c\left(e_{m}\right)$; for, otherwise, by Lemma 3.2, (2) holds. Thus $E(D \cap T)=\emptyset$.

Let $c^{\prime}$ denote the partial edge-coloring obtained from $c$ by interchanging $D$. Since $E(D \cap$ $T)=\emptyset$ and either $D$ is a cycle or both ends of $D$ are contained in $G-T_{m-1}, c^{\prime}\left(e_{i}\right)=c\left(e_{i}\right)$ for $1 \leq i \leq m$, and $M_{c^{\prime}}(v)=M_{c}(v)$ for all $v \in V\left(T_{m-1}\right)$.

If no $e_{i}, i<m$, is connecting in $(T, c)$, then clearly $\left(T_{m-1}, c^{\prime}\right)$ is a VKT-tree without connecting edges. Now suppose that there exists a maximum $s$ such that $s<m$ and $e_{s}$ is a connecting edge in $(T, c)$. Then $c^{\prime}\left(e_{j}\right) \in M_{c^{\prime}}\left(T_{j-1}\right)$ for $s+1 \leq j \leq m$. Since $P_{m}$ contains all edges in $\left[T_{s-1}, G-T_{s-1}\right]$ with the color $c\left(e_{m}\right), D \subseteq G-T_{s-1}$. Hence, by Lemma $3.1,\left(T_{s}, c^{\prime}\right)$ is a VKT-tree in $\left(G, a b, c^{\prime}\right)$, and any edge of $T_{s}$ is connecting in $\left(T_{s}, c^{\prime}\right)$ iff it is connecting in $(T, c)$. By Lemma $2.5(a)$, we see that $\left(T_{m-1}, c^{\prime}\right)$ is a VKT-tree in $\left(G, a b, c^{\prime}\right)$.

Let $e \in E(D) \cap\left[T_{m-1}, G-T_{m-1}\right]$. Then $c^{\prime}(e)=\alpha_{m} \in M_{c^{\prime}}\left(x_{m}\right)$. So by Lemma $2.5(a)$, $\left(T_{m-1}+e, c^{\prime}\right)$ is a VKT-tree in $\left(G, a b, c^{\prime}\right)$. Clearly, $(T, c)<_{2}\left(T_{m-1}+e, c^{\prime}\right)$; and so (2) holds with $T^{\prime}:=T_{m-1}+e$.

The proof of Lemma 3.3 shows that it takes $O(V \Delta)$ time to certify that Lemma 3.3(1) holds or find the $\left(T^{\prime}, c^{\prime}\right)$ in Lemma $3.3(2)$.

The next result is a key lemma, which shows the effect that certain interchanges have on the location of augmenting pairs. This will be used to augment the set of colored edges (through $<_{1}$ ) or to "augment" a VKT-tree (through $\left.<_{2}\right)$. The conditions $\{\alpha, \beta\} \cap M_{c^{\prime}}(x) \cap M_{c^{\prime}}(z) \neq \emptyset$ and $\{\alpha, \beta\} \cap M_{c^{\prime}}(y) \cap M_{c^{\prime}}(z) \neq \emptyset$ in (1) and (3) in the lemma will ensure that when $\chi^{\prime}(G) \geq$ $\Delta(G)+\sqrt{\Delta(G) / 2}$ we can avoid certain colors in our recoloring process. Also note that the VKT-tree $\left(T_{q}, c^{\prime}\right)$ in (1) satisfies $\left(T_{q}, c^{\prime}\right)<_{1}(T, c)$; but it is stated in this explicit form for the convenience of applications (this comment applies to several other lemmas as well).

Lemma 3.4 Let $(G, a b, c)$ be a triple, let $(T, c)$ be a VKT-tree in $(G, a b, c)$ with edge ordering $e_{1}, \ldots, e_{m}$, and let $y$ be the top of $(T, c)$. Let $x \in V\left(T_{t}-T_{t-1}\right)$ and $z \in V\left(T_{s}-T_{s-1}\right)$, where $s \neq t$ and $1 \leq s, t \leq m-1$, and let $q:=\max \{s, t\}$. Suppose $\alpha \in M_{c}(x) \cap M_{c}(y)$ and $\beta \in M_{c}(z)$ such that $\beta \neq \alpha$ and $\{\alpha, \beta\} \cap c\left(T_{q}\right)=\emptyset$. Then one of the following holds.

(1) There exists a component $A$ of $G_{c}(\alpha, \beta)$ such that $|V(A) \cap\{x, z\}|=1$, and if $c^{\prime}$ denotes the partial edge-coloring of $G$ obtained from $c$ by interchanging $A$, then $\{\alpha, \beta\} \cap M_{c^{\prime}}(x) \cap$ $M_{c^{\prime}}(z) \neq \emptyset,\left(T_{q}, c^{\prime}\right)$ is a VKT-tree in $\left(G, a b, c^{\prime}\right)$, and any edge of $T_{q}$ is connecting in $\left(T_{q}, c^{\prime}\right)$ iff it is connecting in $(T, c)$.

(2) There exists a partial edge-coloring $c^{\prime}$ of $G$ obtained from $c$ by an interchange and there is a VKT-tree $\left(T^{\prime}, c^{\prime}\right)$ in $\left(G, a b, c^{\prime}\right)$ such that $(T, c)<_{2}\left(T^{\prime}, c^{\prime}\right)$.

(3) There exists a component $A$ of $G_{c}(\alpha, \beta)$ such that $V(A) \cap\{x, y, z\} \neq \emptyset$ and $\mid V(A) \cap$ $\{x, z\} \mid \neq 1$, and if $c^{\prime}$ denotes the partial edge-coloring of $G$ obtained from $c$ by interchanging $A$, then $\{\alpha, \beta\} \cap M_{c^{\prime}}(y) \cap M_{c^{\prime}}(z) \neq \emptyset,\left(T, c^{\prime}\right)$ is a VKT-tree in $\left(G, a b, c^{\prime}\right)$ with 
edge ordering $e_{1}, \ldots, e_{m}$, and any edge of $T$ is connecting in $\left(T, c^{\prime}\right)$ iff it is connecting in $(T, c)$.

Proof. Let $A$ be a component of $G_{c}(\alpha, \beta)$ intersecting $\{x, y, z\}$, and let $c^{\prime}$ denote the partial edge-coloring of $G$ obtained from $c$ by interchanging $A$. Note that $M_{c^{\prime}}(v)-\{\alpha, \beta\}=M_{c}(v)-$ $\{\alpha, \beta\}$ for all $v \in V(G)$. Hence for any $1 \leq i \leq m, M_{c^{\prime}}\left(T_{i}\right)-\{\alpha, \beta\}=M_{c}\left(T_{i}\right)-\{\alpha, \beta\}$. Since $V(A) \cap\{x, y, z\} \neq \emptyset, A$ is a path, possibly trivial. We claim that

(a) for $1 \leq i \leq q, c\left(e_{i}\right) \in M_{c}\left(T_{i-1}\right)$ iff $c^{\prime}\left(e_{i}\right) \in M_{c^{\prime}}\left(T_{i-1}\right)$, and

(b) if $|A \cap\{x, z\}| \neq 1$ then, for $1 \leq i \leq m, c\left(e_{i}\right) \in M_{c}\left(T_{i-1}\right)$ iff $c^{\prime}\left(e_{i}\right) \in M_{c^{\prime}}\left(T_{i-1}\right)$.

To prove this claim, we choose an arbitrary edge $e_{i}, 1 \leq i \leq m$. First, assume $c\left(e_{i}\right) \notin\{\alpha, \beta\}$. Then $c^{\prime}\left(e_{i}\right)=c\left(e_{i}\right), c\left(e_{i}\right) \in M_{c}\left(T_{i-1}\right)$ iff $c\left(e_{i}\right) \in M_{c}\left(T_{i-1}\right)-\{\alpha, \beta\}$, and $c^{\prime}\left(e_{i}\right) \in M_{c^{\prime}}\left(T_{i-1}\right)$ iff $c^{\prime}\left(e_{i}\right) \in M_{c^{\prime}}\left(T_{i-1}\right)-\{\alpha, \beta\}$. Therefore, since $M_{c}\left(T_{i-1}\right)-\{\alpha, \beta\}=M_{c^{\prime}}\left(T_{i-1}\right)-\{\alpha, \beta\}$, $c\left(e_{i}\right) \in M_{c}\left(T_{i-1}\right)$ iff $c^{\prime}\left(e_{i}\right) \in M_{c^{\prime}}\left(T_{i-1}\right)$. Now assume $c\left(e_{i}\right) \in\{\alpha, \beta\}$. Then $i \geq q+1$ (by assumption). In particular, (a) holds. To see that (b) holds, let us assume $|A \cap\{x, z\}| \neq 1$. Then $\{\alpha, \beta\} \subseteq M_{c^{\prime}}(\{x, z\}) \subseteq M_{c^{\prime}}\left(T_{q}\right)$. Since $i \geq q+1, c^{\prime}\left(e_{i}\right) \in\{\alpha, \beta\} \subseteq M_{c^{\prime}}\left(T_{q}\right) \subseteq M_{c^{\prime}}\left(T_{i-1}\right)$. So $c\left(e_{i}\right) \in M_{c}\left(T_{i-1}\right)$ iff $c^{\prime}\left(e_{i}\right) \in M_{c^{\prime}}\left(T_{i-1}\right)$, completing the proof of this claim.

Since $\{\alpha, \beta\} \cap c\left(T_{q}\right)=\emptyset$ and $\{\alpha, \beta\} \subseteq M_{c}\left(T_{q}\right)$, no connecting edge in $(T, c)$ uses the color $\alpha$ or $\beta$. Hence, if $e_{i}$ is a connecting edge in $(T, c)$, then for any $e \in E(c) \cap\left[T_{i-1}, G-T_{i-1}\right]$, $c(e)=c\left(e_{i}\right)$ implies $c^{\prime}(e)=c(e)$. Thus

(c) $\left(T, c^{\prime}\right)$ with edge ordering $e_{1}, \ldots, e_{m}$ satisfies Definition 2.3(ii)(b).

Next, we show that we may assume that $A$ may be chosen so that

(d) $\left(T, c^{\prime}\right)$ with edge ordering $e_{1}, \ldots, e_{m}$ satisfies Definition 2.3(ii)(c).

Since $(T, c)$ is a VKT-tree in $(G, a b, c)$, for each connecting edge $e_{i}$ there exist $x_{i} \in V_{0}(T, c)$ and $\alpha_{i} \in M_{c}\left(x_{i}\right)-c\left(T_{i}\right)$ such that $G\left[T_{i}\right] \cap G_{c}\left(\alpha_{i}, c\left(e_{i}\right)\right)$ contains a path from $x_{i}$ to $e_{i}$.

Clearly, if $\alpha, \beta \notin M_{c}\left(V_{0}(T, c)\right)$ then, since no connecting edge in $(T, c)$ uses $\alpha$ or $\beta$, for each connecting edge $e_{i}, G\left[T_{i}\right] \cap G_{c^{\prime}}\left(\alpha_{i}, c^{\prime}\left(e_{i}\right)\right)=G\left[T_{i}\right] \cap G_{c}\left(\alpha_{i}, c\left(e_{i}\right)\right)$ contains a path from $x_{i}$ to $e_{i}$; and in this case, we may choose $A$ to be any component of $G_{c}(\alpha, \beta)$ with $V(A) \cap\{x, y, z\} \neq \emptyset$.

If $\{\alpha, \beta\} \subseteq M_{c}\left(V_{0}(T, c)\right)$, then for any connecting edge $e_{i}$, no edge in $\left[T_{i-1}, G-T_{i-1}\right]$ uses $\alpha$ or $\beta$; and hence, we may choose $A$ to be the component of $G_{c}(\alpha, \beta)$ containing $y$ (so $A \subseteq G-T_{i-1}$ whenever $e_{i}$ is a connecting edge, and (d) holds).

Now suppose $\alpha \in M_{c}(v)$ (with $v \in V_{0}(T, c)$ ) and $\beta \notin M_{c}\left(V_{0}(T, c)\right.$ ). By Lemma 3.2, either (2) holds, or for each connecting edge $e_{i}$ in $(T, c)$, the component of $G_{c}(\alpha, \beta)$ containing $v$ contains all edges in $\left[T_{i-1}, G-T_{i-1}\right]$ with color $\beta$. We thus may assume the latter. Then we may choose $A$ to be the component not containing $v$ but containing one of $\{x, y\}$ such that $A \subseteq G-T_{i-1}$ for all connecting edges $e_{i}$; and (d) holds.

The case when $\beta \in M_{c}\left(V_{0}(T, c)\right)$ and $\alpha \notin M_{c}\left(V_{0}(T, c)\right)$ is similar. So we may assume (d).

Having taken care of Definition 2.3(ii)(b) and Definition 2.3(ii) $(c)$ for $\left(T, c^{\prime}\right)$, we now divide the remainder of this proof into two cases.

Case 1. $|A \cap\{x, z\}|=1$. 
Then either $M_{c^{\prime}}(x)=M_{c}(x)$ and $\left(M_{c}(z)-\{\beta\}\right) \cup\{\alpha\} \subseteq M_{c^{\prime}}(z)$, or $M_{c^{\prime}}(z)=M_{c}(z)$ and $\left(M_{c}(x)-\{\alpha\}\right) \cup\{\beta\} \subseteq M_{c^{\prime}}(x)$. Hence, $\{\alpha, \beta\} \cap M_{c^{\prime}}(x) \cap M_{c^{\prime}}(z) \neq \emptyset$.

Clearly, $\left(T_{q}, c^{\prime}\right)$ satisfies Definition 2.3(i). Suppose for all $1 \leq i \leq q$ with $c^{\prime}\left(e_{i}\right) \notin M_{c^{\prime}}\left(T_{i-1}\right)$ and for all $e \in E\left(c^{\prime}\right) \cap\left[T_{i-1}, G-T_{i-1}\right]$, we have $c^{\prime}(e) \notin M_{c^{\prime}}\left(T_{i-1}\right)$. (In fact, we need only to check for those $e$ with $c^{\prime}(e) \in\{\alpha, \beta\}$.) Then $\left(T_{q}, c^{\prime}\right)$ satisfies Definition 2.3(ii)(a). Hence by (c) and $(\mathrm{d}),\left(T_{q}, c^{\prime}\right)$ is a VKT-tree in $\left(G, a b, c^{\prime}\right)$. By (a), any edge of $T_{q}$ is connecting in $\left(T_{q}, c^{\prime}\right)$ iff it connecting in $(T, c)$. So $(1)$ holds.

We may therefore assume that there exist some $i(1 \leq i \leq q)$ and $e \in E\left(c^{\prime}\right) \cap\left[T_{i-1}, G-T_{i-1}\right]$ such that $c^{\prime}\left(e_{i}\right) \notin M_{c^{\prime}}\left(T_{i-1}\right)$ and $c^{\prime}(e) \in M_{c^{\prime}}\left(T_{i-1}\right)$. Choose minimum such $i$. Then by (c) and (d), $\left(T_{i-1}, c^{\prime}\right)$ is a VKT-tree in $\left(G, a b, c^{\prime}\right)$. So by Lemma $2.5(a),\left(T_{i-1}+e, c^{\prime}\right)$ is a VKT-tree in $\left(G, a b, c^{\prime}\right)$, with edge ordering $e_{1}, \ldots, e_{i-1}, e$. By (a), any edge of $T_{i-1}+e$ is connecting in $\left(T_{i-1}+e, c^{\prime}\right)$ iff it is connecting in $(T, c)$. Thus $(T, c)<_{2}\left(T_{i-1}+e, c^{\prime}\right)$. Hence, $(2)$ holds with $T^{\prime}:=T_{i-1}+e$.

Case 2. $|A \cap\{x, z\}| \neq 1$.

When $|A \cap\{x, z\}|=2$, we have $\left(M_{c}(z)-\{\beta\}\right) \cup\{\alpha\}=M_{c^{\prime}}(z)$ and $\left(M_{c}(x)-\{\alpha\}\right) \cup\{\beta\}=$ $M_{c^{\prime}}(x)$, which implies $\alpha \in M_{c^{\prime}}(y) \cap M_{c^{\prime}}(z)$. When $|A \cap\{x, z\}|=0$, we have $y \in A$. In this case, $\left(M_{c}(y)-\{\alpha\}\right) \cup\{\beta\} \subseteq M_{c^{\prime}}(y), M_{c^{\prime}}(z)=M_{c}(z)$, and $M_{c^{\prime}}(x)=M_{c}(x)$, which implies $\beta \in M_{c^{\prime}}(y) \cap M_{c^{\prime}}(z)$. In either case, we have $\{\alpha, \beta\} \cap M_{c^{\prime}}(y) \cap M_{c^{\prime}}(z) \neq \emptyset$.

Clearly, $\left(T, c^{\prime}\right)$ with edge ordering $e_{1}, \ldots, e_{m}$ satisfies Definition 2.3(i). Suppose for all $1 \leq$ $i \leq m$ with $c^{\prime}\left(e_{i}\right) \notin M_{c^{\prime}}\left(T_{i-1}\right)$ and for all $e \in E\left(c^{\prime}\right) \cap\left[T_{i-1}, G-T_{i-1}\right]$ we have $c^{\prime}(e) \notin M_{c^{\prime}}\left(T_{i-1}\right)$. Then $\left(T, c^{\prime}\right)$ satisfies Definition 2.3(ii) (a). So by (c) and (d), $\left(T, c^{\prime}\right)$ is a VKT-tree in $\left(G, a b, c^{\prime}\right)$. By (b), any edge of $T$ is connecting in $\left(T, c^{\prime}\right)$ iff it is connecting in $(T, c)$. Hence, $(3)$ holds.

So we may assume that there exist some $i(1 \leq i \leq m)$ and $e \in E\left(c^{\prime}\right) \cap\left[T_{i-1}, G-T_{i-1}\right]$ such that $c^{\prime}\left(e_{i}\right) \notin M_{c^{\prime}}\left(T_{i-1}\right)$ and $c^{\prime}(e) \in M_{c^{\prime}}\left(T_{i-1}\right)$. (Again, we need only to check for those $e$ with $c^{\prime}(e) \in\{\alpha, \beta\}$.) Choose minimum such $i$. Then by (c) and (d), $\left(T_{i-1}, c^{\prime}\right)$ is a VKT-tree in $\left(G, a b, c^{\prime}\right)$. So by Lemma $2.5(a),\left(T_{i-1}+e, c^{\prime}\right)$ is a VKT-tree in $\left(G, a b, c^{\prime}\right)$, with edge ordering $e_{1}, \ldots, e_{i-1}, e$. By (b), any edge of $T_{i-1}+e$ is connecting in $\left(T_{i-1}+e, c^{\prime}\right)$ iff it is connecting in $(T, c)$. Clearly, $(T, c)<_{2}\left(T_{i-1}+e, c^{\prime}\right)$. Hence, $(2)$ holds with $T^{\prime}:=T_{i-1}+e$.

From the proof of Lemma 3.4, we see that it takes $O(V \Delta)$ time to find the $\left(T_{q}, c^{\prime}\right)$ in Lemma 3.4(1), or the $\left(T^{\prime}, c^{\prime}\right)$ in Lemma 3.4(2), or the $\left(T, c^{\prime}\right)$ in Lemma 3.4(3).

When we apply Lemmas 3.4, we often want to avoid a certain color, say $\gamma$, by choosing $\alpha$ and $\beta$ such that $\gamma \notin\{\alpha, \beta\}$. This will be possible when we have at least $\Delta(G)+\sqrt{\Delta(G) / 2}$ colors; and the next two lemmas will help us achieve this goal.

Lemma 3.5 Let $(G, a b, c)$ be a k-triple with $k \geq \Delta(G)+\sqrt{\Delta(G) / 2}$, let $(T, c)$ be a VKT-tree in $(G, a b, c)$ with edge ordering $e_{1}, \ldots, e_{m}$, and assume that $\left(T_{m-1}, c\right)$ is nonaugmenting. Then

(1) $(T, c)$ has less than $\sqrt{\Delta(G) / 2}$ connecting edges, and

(2) if $2 \leq t \leq m-1$ then $\left|M_{c}\left(T_{t}\right)-c(T)\right| \geq\lfloor\sqrt{\Delta(G) / 2}\rfloor+1$.

Proof. To prove (1), let $s$ denote the number of connecting edges in $(T, c)$. If $s=0$ then (1) holds (note that we assume $\Delta(G) \geq 3$ ). So we may assume $s \geq 1$. Since $\left(T_{m-1}, c\right)$ is nonaugmenting, each segment of $(T, c)$ must be incident with at least three vertices, except possibly the last segment which is incident with at least two vertices. So $\left|V\left(T_{m-1}\right)\right| \geq 2 s+1$. 
Therefore, $k \geq\left|M_{c}\left(T_{m-1}\right)\right|>(2 s+1)(k-\Delta(G))$ (since $a b$ is not colored and $\left(T_{m-1}, c\right)$ is nonaugmenting). This implies that $k<\Delta(G)+\Delta(G) /(2 s)$. If $s \geq \sqrt{\Delta(G) / 2}$ then $k<$ $\Delta(G)+\sqrt{\Delta(G) / 2}$, a contradiction. So $s<\sqrt{\Delta(G) / 2}$, and (1) holds.

Suppose (2) fails. Then all but at most $\sqrt{\Delta(G) / 2}$ colors in $M_{c}\left(T_{t}\right)$ are used by some edge in $(T, c)$. Hence, since $\left(T_{m-1}, c\right)$ (and thus $\left.\left(T_{t}, c\right)\right)$ is nonaugmenting and $a b$ is not colored,

$$
m-1 \geq(t+1)(k-\Delta(G))+2-\sqrt{\Delta(G) / 2} \geq 3(k-\Delta(G))+2-\sqrt{\Delta(G) / 2}
$$

and

$$
\left|M_{c}\left(T_{m-1}\right)\right| \geq m(k-\Delta(G))+2 .
$$

So

$$
k \geq\left|M_{c}\left(T_{m-1}\right)\right| \geq(3(k-\Delta(G))+3-\sqrt{\Delta(G) / 2})(k-\Delta(G))+2 .
$$

Therefore, $3(k-\Delta(G))^{2}-(\sqrt{\Delta(G) / 2}-2)(k-\Delta(G))-(\Delta(G)-2) \leq 0$. Solving for $k-\Delta(G)$, we deduce $k-\Delta(G)<\sqrt{\Delta(G) / 2}$, a contradiction.

Note that it takes $O(V \Delta)$ time to find a vertex $y$ of $T_{t}$ and a color $\beta \in M_{c}(y)-c(T)$.

Lemma 3.6 Let $(G, a b, c)$ be a k-triple, let $(T, c)$ be a VKT-tree in $(G, a b, c)$ with edge ordering $e_{1}, \ldots, e_{m}$, and assume that $\left(T_{m-1}, c\right)$ is nonaugmenting. Let $x \in V\left(T_{t+1}-T_{t}\right)$ where $1 \leq t \leq$ $m-2$. Then

(1) if $\alpha \in M_{c}(x)$ and $\alpha$ is not used by any connecting edge in $(T, c)$, then $\alpha \notin c\left(T_{t+1}\right)$, and

(2) if $k \geq \Delta(G)+\sqrt{\Delta(G) / 2}$ then $M_{c}(x)-c\left(T_{t+1}\right) \neq \emptyset$.

Proof. To prove (1), let us assume for a contradiction that there exists some $s, 1 \leq s \leq t+1$, such that $c\left(e_{s}\right)=\alpha$. Clearly, $s \neq 1$ and $s \neq t+1$. Hence $2 \leq s \leq t$. Since $\alpha$ is not used by any connecting edge in $(T, c), \alpha=c\left(e_{s}\right) \in M_{c}\left(T_{s-1}\right)$. Let $z \in V\left(T_{s-1}\right)$ such that $\alpha \in M_{c}(z)$. Then $\{x, z\}$ is an augmenting pair in $\left(T_{m-1}, c\right)$, a contradiction.

Now suppose $M_{c}(x) \subseteq c\left(T_{t+1}\right)$. Then by (1), each edge of $T_{t+1}$ with color $\alpha \in M_{c}(x)$ must be a connecting edge in $(T, c)$. Therefore, there are at least $k-\Delta(G) \geq \sqrt{\Delta(G) / 2}$ connecting edges in $(T, c)$, contradicting Lemma 3.5(1).

Note that for Lemma 3.6(2), it takes $O(V \Delta)$ time to find a color in $M_{c}(x)-c\left(T_{t+1}\right)$.

\section{Working with augmenting pairs}

In this section, we prove lemmas concerning VKT-trees with augmenting pairs. First, we show how to produce a good augmenting pair from any given augmenting pair.

Lemma 4.1 Let $(G, a b, c)$ be a $k$-triple with $k \geq \Delta(G)+\sqrt{\Delta(G) / 2}$, let $(T, c)$ be a VKT-tree in $(G, a b, c)$ with edge ordering $e_{1}, \ldots, e_{m}$, and let $y$ be the top of $(T, c)$. Assume $m \geq 2$, $\left(T_{m-1}, c\right)$ is nonaugmenting, and $y$ is in an augmenting pair in $(T, c)$. Then there exists a partial $k$-edge-coloring $c^{\prime}$ of $G$ obtained from $c$ by an interchange such that one of the following holds. 
(1) There exists an integer $t, 1 \leq t \leq m$, such that $\left(T_{t}, c^{\prime}\right)$ is a VKT-tree in $\left(G, a b, c^{\prime}\right)$, $\left(T_{t-1}, c^{\prime}\right)$ is nonaugmenting, the top of $T_{t}$ is contained in a good augmenting pair in $\left(T_{t}, c^{\prime}\right)$, and any edge of $T_{t}$ is connecting in $\left(T_{t}, c^{\prime}\right)$ iff it is connecting in $(T, c)$.

(2) There is a VKT-tree $\left(T^{\prime}, c^{\prime}\right)$ in $\left(G, a b, c^{\prime}\right)$ such that $(T, c)<_{2}\left(T^{\prime}, c^{\prime}\right)$.

Proof. Let $x \in V\left(T_{t}-T_{t-1}\right)(1 \leq t \leq m-1)$ such that $\{x, y\}$ is an augmenting pair in $(T, c)$. Let $\alpha \in M_{c}(y) \cap M_{c}(x)$. We may assume that $\alpha$ is used by some connecting edge in $(T, c)$, as otherwise (1) holds with $t:=m$ and $c^{\prime}:=c$. In particular, $\alpha \in c(T)$. Note that $\left|V_{0}(T, c)\right| \geq 3$, because $\left(T_{m-1}, c\right)$ is nonaugmenting. So by Lemma 3.5(2), there exist $z \in V_{0}(T, c)$ and $\beta \in M_{c}(z)-(c(T) \cup\{\alpha\})$. Let $X, Y, Z$ denote the components of $G_{c}(\alpha, \beta)$ containing $x, y, z$, respectively.

We may assume that whenever $e_{i}$ is a connecting edge, $Z$ contains those edges in $\left[T_{i-1}, G-\right.$ $T_{i-1}$ ] that use color $\alpha$. Otherwise, by Lemma 3.2, (2) holds.

Suppose $z \notin V(X)$. In this case, $X \subseteq G-T_{i-1}$ whenever $i \leq t$ and $e_{i}$ is a connecting edge in $(T, c)$. (Such $e_{i}$ exists, since $\alpha \in M_{c}(x)$ and $\alpha$ is used by a connecting edge in $(T, c)$.) In particular, let $p \leq t$ with $p$ maximum such that $e_{p}$ is a connecting edge in $(T, c)$; then $X \subseteq G-T_{p-1}$. Let $c^{\prime}$ denote the partial $k$-edge coloring of $G$ obtained from $c$ by interchanging $X$. By Lemma 3.1, $\left(T_{p}, c^{\prime}\right)$ is a VKT-tree in $G$ with edge ordering $e_{1}, \ldots, e_{p}$, and any edge of $T_{p}$ is connecting in $\left(T_{p}, c^{\prime}\right)$ iff it is connecting in $(T, c)$. For $p<j \leq t$, we have $c\left(e_{j}\right) \in M_{c}\left(T_{j-1}\right)$. Since $\beta \notin c(T)$ and $\alpha \in M_{c}(x)-M_{c}\left(T_{t-1}\right)$, we see that $c\left(e_{j}\right) \notin\{\alpha, \beta\}$; and hence, $c^{\prime}\left(e_{j}\right) \in M_{c^{\prime}}\left(T_{j-1}\right)$. Therefore, by repeatedly applying Lemma $2.5(a),\left(T_{t}, c^{\prime}\right)$ is a VKT-tree in $G$ with edge ordering $e_{1}, \ldots, e_{t}$, and any edge of $T_{t}$ is connecting in $\left(T_{t}, c^{\prime}\right)$ iff it is connecting in $(T, c)$. Moreover, $\left(T_{t-1}, c^{\prime}\right)$ is nonaugmenting, and $\beta \in M_{c^{\prime}}(x) \cap M_{c^{\prime}}(z)$. So (1) holds, since $\beta$ is not used by any connecting edge in $\left(T_{t}, c^{\prime}\right)$.

We thus may assume $z \in V(X)$. Then $z \notin V(Y)$. Hence, $Y \subseteq G-T_{i-1}$ whenever $e_{i}$ is a connecting edge in $(T, c)$. So let $e_{p}$ be the last connecting edge in $(T, c)$; then $Y \subseteq G-T_{p-1}$. Let $c^{\prime}$ denote the partial $k$-edge coloring of $G$ obtained from $c$ by interchanging $Y$. By Lemma 3.1, $\left(T_{p}, c^{\prime}\right)$ is a VKT-tree in $\left(G, a b, c^{\prime}\right)$, and any edge of $T_{p}$ is connecting in $\left(T_{p}, c^{\prime}\right)$ iff it is connecting in $(T, c)$. Note that $\alpha \in M_{c^{\prime}}(x)$ and $\beta \in M_{c^{\prime}}(z)$. So for $p<j \leq t, c^{\prime}\left(e_{j}\right) \in M_{c^{\prime}}\left(T_{j-1}\right)$. It then follows from Lemma $2.5(a)$ that $\left(T, c^{\prime}\right)$ is a VKT-tree in $\left(G, a b, c^{\prime}\right)$. Since $\left(T_{m-1}, c\right)$ is nonaugmenting, the end of $Y$ other than $y$ is not in $T$; so $\left(T_{m-1}, c^{\prime}\right)$ is also nonaugmenting. Now $\beta \in M_{c^{\prime}}(y) \cap M_{c^{\prime}}(z)$ and $\beta$ is not used by any connecting edge in $\left(T, c^{\prime}\right)$. Hence, $y$ is contained in a good augmenting pair, and (1) holds.

From the proof, we see that given the augmenting pair $\{x, y\}$, it takes $O(V \Delta)$ time to find the $\left(T_{t}, c^{\prime}\right)$ in Lemma $4.1(1)$ or the $\left(T^{\prime}, c^{\prime}\right)$ in Lemma 4.1(2).

The next lemma roughly says that if $\{x, y\}$ is a divided augmenting pair in $(T, c)$, then $(T, c)$ can be augmented with respect to the partial order $<_{2}$, or $c$ can be modified to $c^{\prime}$ so that $\left(T, c^{\prime}\right)$ has an undivided augmenting pair.

Lemma 4.2 Let $(G, a b, c)$ be a k-triple with $k \geq \Delta(G)+\sqrt{\Delta(G) / 2}$, let $(T, c)$ be a VKT-tree in $(G, a b, c)$ with edge ordering $e_{1}, \ldots, e_{m}$, let $y$ be the top of $(T, c)$, and assume that $m \geq 2$ and $\left(T_{m-1}, c\right)$ is nonaugmenting. Let $e_{s}$ be the last connecting edge in $(T, c)$, let $y_{s}$ be the end of $e_{s}$ with $y_{s} \notin T_{s-1}$, and assume that $y$ and a vertex of $T_{s-1}$ form a good augmenting pair in $(T, c)$. Then there exists a partial $k$-edge-coloring $c^{\prime}$ of $G$ obtained from $c$ by a sequence of at most two interchanges such that one of the following holds. 
(1) $\left(T, c^{\prime}\right)$ is a VKT-tree in $\left(G, a b, c^{\prime}\right)$ with edge ordering $e_{1}, \ldots, e_{m},\left(T_{m-1}, c^{\prime}\right)$ is nonaugmenting, $\left\{y_{s}, y\right\}$ is an undivided, good, augmenting pair in $\left(T, c^{\prime}\right)$, and any edge of $T$ is connecting in $\left(T, c^{\prime}\right)$ iff it is connecting in $(T, c)$.

(2) There is a VKT-tree $\left(T^{\prime}, c^{\prime}\right)$ in $\left(G, a b, c^{\prime}\right)$ such that $(T, c)<_{2}\left(T^{\prime}, c^{\prime}\right)$.

Proof. Let $x \in V\left(T_{s-1}\right)$ and $\alpha \in M_{c}(x) \cap M_{c}(y)$ such that $\alpha$ is not used by any connecting edge in $(T, c)$. Note that $s \geq 3$, since $V_{0}(T, c)$ is nonaugmenting. We may assume $s<m$, as otherwise (2) holds by Lemma 3.3.

Claim. We may assume that $x \in V_{0}(T, c)$ and $\alpha \notin c\left(T_{s}\right)$.

By Lemma 3.5(2), there exist $w \in V_{0}(T, c)$ and $\gamma \in M_{c}(w)-c(T)$. If $\gamma \in M_{c}(y)$, then this claim holds by taking $x:=w$ and $\alpha:=\gamma$. So we may assume $\gamma \notin M_{c}(y)$; in particular, $\alpha \neq \gamma$.

Let $Y$ denote the component of $G_{c}(\alpha, \gamma)$ containing $y$; then $Y \subseteq G-T_{s-1}$ (because no edge in $\left[T_{s-1}, G-T_{s-1}\right]$ uses the color $\alpha$ or $\left.\gamma\right)$. Since $\left(T_{m-1}, c\right)$ is nonaugmenting, the end of $Y$ other than $y$ is not in $T$. Let $c^{*}$ denote the partial $k$-edge-coloring of $G$ obtained from $c$ by interchanging $Y$. Then by Lemma $3.1,\left(T_{s}, c^{*}\right)$ is a VKT-tree in $\left(G, a b, c^{*}\right)$, and any edge of $T_{s}$ is connecting in $\left(T_{s}, c^{*}\right)$ iff it is connecting in $(T, c)$.

Note that $M_{c^{*}}(v)=M_{c}(v)$ for all $v \in V(T-y), \gamma \in M_{c^{*}}(y) \cap M_{c^{*}}(w)$, and $\gamma \notin c^{*}\left(T_{s}\right)$.

For any $s+1 \leq j \leq m$, if $c\left(e_{j}\right) \notin\{\alpha, \gamma\}$ then $c^{*}\left(e_{j}\right)=c\left(e_{j}\right) \in M_{c}\left(T_{j-1}\right)=M_{c^{*}}\left(T_{j-1}\right)$ (because $e_{s}$ is the last connecting edge in $(T, c)$ ), and if $c\left(e_{j}\right) \in\{\alpha, \gamma\}$ then $c^{*}\left(e_{j}\right) \in\{\alpha, \gamma\} \subseteq$ $M_{c^{*}}(\{x, w\}) \subseteq M_{c^{*}}\left(T_{j-1}\right)$. Hence, by repeatedly applying Lemma $2.5(a),\left(T, c^{*}\right)$ is a VKTtree in $G$ with edge ordering $e_{1}, \ldots, e_{m}$, and $e_{s}$ is also the last connecting edge in $\left(T, c^{*}\right)$. Since $\left(T_{m-1}, c\right)$ is nonaugmenting and $M_{c^{*}}(v)=M_{c}(v)$ for all $v \in V(T-y),\left(T_{m-1}, c^{*}\right)$ is nonaugmenting. So this claim holds by taking $c:=c^{*}$ and $\alpha:=\gamma$.

By Lemma 3.6(2), there exists $\beta \in M_{c}\left(y_{s}\right)-c\left(T_{s}\right)$. Since $\left(T_{m-1}, c\right)$ is nonaugmenting and $s<m, \beta \neq \alpha$. Let $X, Y_{s}, Y$ denote the components of $G_{c}(\alpha, \beta)$ containing $x, y_{s}, y$, respectively. We may assume that there exists $A \in\left\{Y_{s}, Y\right\}$ such that $A \subseteq G-T_{s-1}$; otherwise, (2) holds by Lemma 3.2 and the above claim.

Let $c^{\prime}$ denote the partial edge-coloring of $G$ obtained from $c$ by interchanging $A$. Then by Lemma 3.1, $\left(T_{s}, c^{\prime}\right)=\left(T_{s}, c\right)$ is a VKT-tree in $\left(G, a b, c^{\prime}\right)$, and any edge of $T_{s}$ is connecting in $\left(T_{s}, c^{\prime}\right)$ iff it is connecting in $(T, c)$.

If $A=Y_{s}$ then $\alpha \in M_{c^{\prime}}\left(y_{s}\right) \cap M_{c^{\prime}}(x)$ and $\left(T_{s-1}, c^{\prime}\right)$ is nonaugmenting, and (2) holds by Lemma 3.3. So we may assume $y_{s} \notin A$. Then $y \in A$. Note that $\beta \in M_{c^{\prime}}(y) \cap M_{c^{\prime}}\left(y_{s}\right)$ and $\{\alpha, \beta\} \subseteq M_{c^{\prime}}\left(\left\{x, y_{s}\right\}\right)$. Let $s+1 \leq i \leq m$. Since $e_{s}$ is the last connecting edge in $(T, c)$, if $c\left(e_{i}\right) \notin\{\alpha, \beta\}$ then $c^{\prime}\left(e_{i}\right)=c\left(e_{i}\right) \in M_{c}\left(T_{i-1}\right)-\{\alpha, \beta\}=M_{c^{\prime}}\left(T_{i-1}\right)-\{\alpha, \beta\}$. On the other hand, if $c\left(e_{i}\right) \in\{\alpha, \beta\}$ then $c^{\prime}\left(e_{i}\right) \in M_{c^{\prime}}\left(\left\{x, y_{s}\right\}\right) \subseteq M_{c^{\prime}}\left(T_{i-1}\right)$. Hence by repeatedly applying Lemma 2.5(a) (starting from $\left(T_{s}, c^{\prime}\right)$ ), we see that $\left(T, c^{\prime}\right)$ is a VKT-tree in $\left(G, a b, c^{\prime}\right)$ with edge ordering $e_{1}, \ldots, e_{m}$. Moreover, any edge of $T$ is connecting in $\left(T, c^{\prime}\right)$ iff it is connecting in $(T, c)$. Since $\left(T_{m-1}, c\right)$ is nonaugmenting and $Y \neq Y_{s}$, the end of $A$ other than $y$ is not in $T$. So $\left(T_{m-1}, c^{\prime}\right)$ is also nonaugmenting, and (1) holds.

From the proof of Lemma 4.2, we see that given the good augmenting pair $\{x, y\}$ it takes $O(V \Delta)$ time to find the $\left(T, c^{\prime}\right)$ in Lemma 4.2(1), or the $\left(T^{\prime}, c^{\prime}\right)$ in Lemma 4.2(2).

The following lemma considers VKT-trees $(T, c)$ in which $B(T, c)-C(T, c)$ contains a good augmenting pair. 
Lemma 4.3 Let $(G, a b, c)$ be a k-triple with $k \geq \Delta(G)+\sqrt{\Delta(G) / 2}$, let $(T, c)$ be a VKTtree in $(G, a b, c)$ with edge ordering $e_{1}, \ldots, e_{m}$, let $y$ be the top of $(T, c)$, and let $e_{t+1}=x z$ $(1 \leq t \leq m-1)$ be an edge of $B(T, c)-C(T, c)$ with $x \in T_{t}$ and $z \notin T_{t}$. Assume that $\left(T_{m-1}, c\right)$ is nonaugmenting, and $\{x, y\}$ is a good augmenting pair in $(T, c)$. Then there exists a partial $k$-edge-coloring $c^{\prime}$ of $G$ obtained from $c$ by an interchange such that one of the following holds.

(1) There exists a VKT-tree $\left(T^{\prime}, c^{\prime}\right)$ in $\left(G, a b, c^{\prime}\right)$ with top $y^{\prime}$ such that $\left(T^{\prime}, c^{\prime}\right)<_{1}(T, c)$, $\left(T^{\prime}-y^{\prime}, c^{\prime}\right)$ is nonaugmenting, $y^{\prime}$ is contained in a good augmenting pair in $\left(T^{\prime}, c^{\prime}\right)$, $S\left(T^{\prime}, c^{\prime}\right)$ is a truncation of $S(T, c)$, and any edge of $T^{\prime}$ is connecting in $\left(T^{\prime}, c^{\prime}\right)$ iff it is connecting in $(T, c)$.

(2) There is a VKT-tree $\left(T^{\prime}, c^{\prime}\right)$ in $\left(G, a b, c^{\prime}\right)$ such that $(T, c)<_{2}\left(T^{\prime}, c^{\prime}\right)$.

(3) $\left(T, c^{\prime}\right)$ is a VKT-tree in $\left(G, a b, c^{\prime}\right)$ with edge ordering $e_{1}, \ldots, e_{m},\left(T_{m-1}, c^{\prime}\right)$ is nonaugmenting, $\{y, z\}$ is a good augmenting pair in $\left(T, c^{\prime}\right)$, and any edge of $T$ is connecting in $\left(T, c^{\prime}\right)$ iff it is connecting in $(T, c)$.

Proof. Let $C(T, c)=T_{r}$, where $0 \leq r \leq m-1$ and $T_{0}=\emptyset$. Then $B(T, c)=T\left[e_{r+1}, \ldots, e_{m}\right]$. Since $e_{t+1} \in B(T, c)-C(T, c), t \geq r+1$. Since $\{x, y\}$ is a good augmenting pair in $(T, c)$, there exists $\alpha \in M_{c}(x) \cap M_{c}(y)$ such that $\alpha$ is not used by any connecting edge in $(T, c)$. Because $\left(T_{m-1}, c\right)$ is nonaugmenting, it follows from Lemma 3.6(1) that $\alpha \notin c\left(T_{t}\right)$.

We may assume $e_{m}$ is not a connecting edge in $(T, c)$. Otherwise, (2) holds by Lemma 3.3.

Suppose $e_{m}=x y$. Let $\beta:=c\left(e_{m}\right)$. Since $e_{m}$ is not a connecting edge and $\{x, y\} \cap$ $V(C(T, c))=\emptyset, \beta \in M_{c}\left(T_{m-2}\right)$. Let $A$ be the component of $G_{c}(\alpha, \beta)$ that is induced by $e_{m}$, and let $c^{\prime}$ be the partial $k$-edge-coloring of $G$ obtained from $c$ by interchanging $A$. Note that $\beta \in M_{c^{\prime}}(x) \cap M_{c^{\prime}}\left(T_{m-2}\right)$ and $M_{c^{\prime}}(v)=M_{c}(v)$ for all $v \in V\left(T_{m-2}\right)$. Also note that $A \subseteq G-T_{m-2}$. So by Lemma 3.1, $\left(T_{m-1}, c^{\prime}\right)$ is a VKT-tree in $\left(G, a b, c^{\prime}\right)$, and any edge of $T_{m-1}$ is connecting in $\left(T_{m-1}, c^{\prime}\right)$ iff it is connecting in $(T, c)$. Clearly, $\left(T_{m-1}, c^{\prime}\right)<_{1}(T, c)$. Note that $\left(T_{m-2}, c^{\prime}\right)$ is nonaugmenting, and $S\left(T_{m-1}, c^{\prime}\right)$ is a truncation of $S(T, c)$. Hence (1) holds with $T^{\prime}:=T_{m-1}$ and $y^{\prime}:=x$.

Now suppose $e_{m} \neq x y$. Then $m \geq t+2$ and $z \neq y$. By Lemma 3.6(2), we may choose $\beta \in M_{c}(z)-c\left(T_{t+1}\right)$. Since $\left(T_{m-1}, c\right)$ is nonaugmenting, $\beta \neq \alpha$. Note that $c\left(e_{t+1}\right) \notin\{\alpha, \beta\}$. So $\{\alpha, \beta\} \cap c\left(T_{t+1}\right)=\emptyset$. Therefore, we may apply Lemma 3.4 with $q:=t+1$.

If Lemma 3.4(2) or Lemma 3.4(3) holds, we have (2) or (3), respectively. So we may assume Lemma 3.4(1) holds. Then $\left(T_{t+1}, c^{\prime}\right)$ is a VKT-tree in $\left(G, a b, c^{\prime}\right),\{x, z\}$ is a good augmenting pair in $\left(T_{t+1}, c^{\prime}\right)$, and any edge of $T_{t+1}$ is connecting in $\left(T_{t+1}, c^{\prime}\right)$ iff it is connecting in $\left(T_{t+1}, c\right)$. Clearly, $\left(T_{t+1}, c^{\prime}\right)<_{1}(T, c)$. Since $M_{c^{\prime}}(v)=M_{c}(v)$ for all $v \in V\left(T_{t-1}\right)$ and $\{\alpha, \beta\} \cap M_{c}\left(T_{t-1}\right)=\emptyset,\left(T_{t}, c^{\prime}\right)$ is nonaugmenting. So (1) holds with $T^{\prime}:=T_{t+1}$.

The proof of Lemma 4.3 shows that it takes $O(V \Delta)$ time to find the $\left(T^{\prime}, c^{\prime}\right)$ in Lemma 4.3(1), the $\left(T^{\prime}, c^{\prime}\right)$ in Lemma $4.3(2)$, or the $\left(T, c^{\prime}\right)$ in Lemma 4.3(3).

The final case we need to consider is when there is a good augmenting pair in $(T, c)$ that consists of the top of $(T, c)$ and a vertex of $C(T, c)$.

Lemma 4.4 Let $(G, a b, c)$ be a k-triple with $k \geq \Delta(G)+\sqrt{\Delta(G) / 2}$, let $(T, c)$ be a VKT-tree in $(G, a b, c)$ with edge ordering $e_{1}, \ldots, e_{m}$, let $y$ be the top of $(T, c)$, and let $x \in V(C(T, c))$. Assume that $\left(T_{m-1}, c\right)$ is nonaugmenting, and $\{x, y\}$ is a good augmenting pair in $(T, c)$. 
Then there is a partial $k$-edge-coloring $c^{\prime}$ of $G$ obtained from $c$ by a sequence of at most seven interchanges such that one of the following holds.

(1) There exists a VKT-tree $\left(T^{\prime}, c^{\prime}\right)$ in $\left(G, a b, c^{\prime}\right)$ with top $y^{\prime}$ such that $\left(T^{\prime}, c^{\prime}\right)<_{1}(T, c)$, $\left(T^{\prime}-y^{\prime}, c^{\prime}\right)$ is nonaugmenting, $y^{\prime}$ is contained in a good augmenting pair in $\left(T^{\prime}, c^{\prime}\right)$, $S\left(T^{\prime}, c^{\prime}\right)$ is a truncation of $S(T, c)$, and any edge of $T^{\prime}$ is connecting in $\left(T^{\prime}, c^{\prime}\right)$ iff it is connecting in $(T, c)$.

(2) There is a VKT-tree $\left(T^{\prime}, c^{\prime}\right)$ in $\left(G, a b, c^{\prime}\right)$ such that $(T, c)<_{2}\left(T^{\prime}, c^{\prime}\right)$.

(3) $\left(T, c^{\prime}\right)$ is a VKT-tree in $G$ with edge ordering $e_{1}, \ldots, e_{m},\left(T-y, c^{\prime}\right)$ is nonaugmenting, $y$ and a vertex of $B\left(T, c^{\prime}\right)-C\left(T, c^{\prime}\right)$ form a good augmenting pair in $\left(T, c^{\prime}\right)$, and any edge of $T$ is connecting in $\left(T, c^{\prime}\right)$ iff it is connecting in $(T, c)$.

Proof. Let $x \in V\left(T_{t}-T_{t-1}\right)$ and $C(T, c)=T_{r}$, where $t \leq r \leq m-1$. Then $B(T, c)=$ $T\left[e_{r+1}, \ldots, e_{m}\right]$. Note that $r \geq 2$, since $C(T, c) \neq \emptyset$. Let $w$ be the end of $e_{r}$ with $w \notin T_{r-1}$. Since $\{x, y\}$ is a good augmenting pair, there exists $\alpha \in M_{c}(x) \cap M_{c}(y)$ such that $\alpha$ is not used by any connecting edge in $(T, c)$. Then since $\left(T_{m-1}, c\right)$ is nonaugmenting, it follows from Lemma 3.6(1) that $\alpha \notin c\left(T_{t}\right)$.

Claim 1. We may assume $x \in T_{r-1}$, i.e., $x \neq w$.

It suffices to show that (1) or (2) or Claim 1 holds. (This may require one interchange.)

If $x \neq w$ then Claim 1 holds. So we may assume $x=w$; hence $r=t$. By Lemma 3.5(2) (when $r \geq 3$ ), there exist $z \in V\left(T_{r-1}\right) \cap V_{0}(T, c)$ and $\beta \in M_{c}(z)-\left(c\left(T_{r+1}\right) \cup\{\alpha\}\right)$. This also holds when $r=2$, since $\Delta(G) \geq 3$ (which we assume) and $k \geq \Delta(G)+2$. Hence $\{\alpha, \beta\} \cap c\left(T_{r}\right)=\emptyset$, and we may apply Lemma 3.4 (with $q:=r$ ).

If Lemma 3.4(2) holds, then (2) holds.

Suppose Lemma 3.4(1) holds. Then there is a component $A$ of $G_{c}(\alpha, \beta)$ such that $\mid V(A) \cap$ $\{w, z\} \mid=1$, and if $c^{\prime}$ denotes the partial $k$-edge-coloring of $G$ obtained from $c$ by interchanging $A$, then $\left(T_{r}, c^{\prime}\right)$ is a VKT-tree in $G,\{w, z\}$ is a good augmenting pair in $\left(T_{r}, c^{\prime}\right), S\left(T_{r}, c^{\prime}\right)$ is a truncation of $S(T, c)$, and any edge of $T_{r}$ is connecting in $\left(T_{r}, c^{\prime}\right)$ iff it is connecting in $(T, c)$. Moreover, $c^{\prime}\left(e_{i}\right)=c\left(e_{i}\right)$ for all $1 \leq i \leq r$, and $M_{c^{\prime}}(v)=M_{c}(v)$ for all $v \in V\left(T_{r-1}\right)$ except possibly $M_{c^{\prime}}(z)=\left(M_{c}(z)-\{\beta\}\right) \cup\{\alpha\}$. Hence, since $\left(T_{m-1}, c\right)$ is nonaugmenting, $\left(T_{r-1}, c^{\prime}\right)$ is nonaugmenting. So (1) holds with $T^{\prime}:=T_{r}$.

We thus may assume that Lemma 3.4(3) holds. Then there is a component $A$ of $G_{c}(\alpha, \beta)$ intersecting $\{w, y, z\}$ such that $|V(A) \cap\{w, z\}| \neq 1$, and if $c^{\prime}$ denotes the partial $k$-edgecoloring of $G$ obtained from $c$ by interchanging $A$, then $\{\alpha, \beta\} \cap M_{c^{\prime}}(y) \cap M_{c^{\prime}}(z) \neq \emptyset,\left(T, c^{\prime}\right)$ is a VKT-tree in $\left(G, a b, c^{\prime}\right)$, and any edge of $T$ is connecting in $\left(T, c^{\prime}\right)$ iff it is connecting in $(T, c)$. In particular, since $|V(A) \cap\{w, z\}| \neq 1$ and $\left(T_{m-1}, c\right)$ is nonaugmenting, $\left(T_{m-1}, c^{\prime}\right)$ is nonaugmenting. If $|V(A) \cap\{w, z\}|=2$ then $\alpha \in M_{c^{\prime}}(y) \cap M_{c^{\prime}}(z)$ and $\alpha$ is not used by any connecting edge in $\left(T, c^{\prime}\right)$; and if $|V(A) \cap\{w, z\}|=0$ then $\beta \in M_{c^{\prime}}(y) \cap M_{c^{\prime}}(z)$ and $\beta$ is not used by any connecting edge in $\left(T, c^{\prime}\right)$. So $\{y, z\}$ is a good augmenting pair in $\left(T, c^{\prime}\right)$; and we have Claim 1 by taking $c^{\prime}, z$ as $c, x$, respectively.

Claim 2. We may assume that for any $r+1 \leq j \leq m, e_{j}$ is not a connecting edge in $(T, c)$.

For, otherwise, let $e_{s}$ denote the last connecting edge in $(T, c)$ where $r+1 \leq s \leq m$, and let $y_{s} \in V\left(T_{s}-T_{s-1}\right)$. We may assume that $e_{m}$ is not a connecting edge, as otherwise (2) 
holds by Lemma 3.3. So $s<m$. Since $x \in T_{s-1}$, we may apply Lemma 4.2. If Lemma 4.2(1) holds then (3) holds; and if Lemma 4.2(2) holds then (2) holds. (Note that Lemma 4.2 may require two interchanges.)

Claim 3. We may further assume $x \in V_{0}(T, c) \cap V\left(T_{r-1}\right)$ and $\alpha \notin c\left(T_{r+1}\right)$.

It suffices to show that (1) or Claim 3 holds. (This may require one interchange.)

As in the proof of Claim 1, there exist $z \in V\left(T_{r-1}\right) \cap V_{0}(T, c)$ and $\beta \in M_{c}(z)-\left(c\left(T_{r+1}\right) \cup\right.$ $\{\alpha\})$. By Claim $2, \beta$ is not used by any connecting edge in $(T, c)$. So if $\beta \in M_{c}(y)$, then Claim 3 holds by choosing $z, \beta$ as $x, \alpha$, respectively. So we may assume $\beta \notin M_{c}(y)$.

Let $Y$ denote the component of $G_{c}(\alpha, \beta)$ containing $y$. If $Y \cap T_{r}=\emptyset$, let $Y^{\prime}:=Y$ and let $y^{*}$ be the end of $Y$ other than $y$; otherwise, let $Y^{\prime}$ denote the subpath of $Y$ from $y$ to $y^{*} \in V\left(T_{r}\right)$ such that $V\left(Y^{\prime} \cap T_{r}\right)=\left\{y^{*}\right\}$.

Suppose $Y \cap T_{r} \neq \emptyset$ and $y^{*}=w$ (respectively, $\left.y^{*} \in T_{r-1}\right)$. Note that $\{\alpha, \beta\} \subseteq M_{c}\left(T_{r-1}\right)$ (by Claim 1) and each edge of $Y^{\prime}$ uses the color $\alpha$ or $\beta$. Since $\left(T_{r}, c\right)$ (respectively, $\left(T_{r-1}, c\right)$ ) is a VKT-tree in $(G, a b, c)$, it follows from repeated applications of Lemma 2.5(a) that $\left(T_{r} \cup\right.$ $\left.Y^{\prime}, c\right)$ (respectively, $\left(T_{r-1} \cup Y^{\prime}, c\right)$ ) is a VKT-tree in $(G, a b, c)$ with edge ordering $e_{1}, \ldots, e_{r}$ (respectively, $e_{1}, \ldots, e_{r-1}$ ) followed by the edges of $Y^{\prime}$ in order from $y^{*}$ to $y$. Clearly, $C\left(T_{r} \cup\right.$ $\left.Y^{\prime}, c\right) \subseteq T_{r-1}$ (respectively, $C\left(T_{r-1} \cup Y^{\prime}, c\right) \subseteq T_{r-1}$ ), which is properly contained in $C(T, c)=$ $T_{r}$. So $\left(T_{r} \cup Y^{\prime}, c\right)<_{1}(T, c)$ (respectively, $\left.\left(T_{r-1} \cup Y^{\prime}, c\right)<_{1}(T, c)\right)$. Moreover, no edge of $Y^{\prime}$ is connecting in $\left(T_{r} \cup Y^{\prime}, c\right)$ (respectively, $\left(T_{r-1} \cup Y^{\prime}, c\right)$ ). Hence, $S\left(T_{r} \cup Y^{\prime}, c\right)$ (respectively, $\left.S\left(T_{r-1} \cup Y^{\prime}, c\right)\right)$ is a truncation of $S(T, c)$, and any edge of $T_{r} \cup Y^{\prime}$ (respectively, $T_{r-1} \cup Y^{\prime}$ ) is connecting in $\left(T_{r} \cup Y^{\prime}, c\right)$ (respectively, $\left(T_{r-1} \cup Y^{\prime}, c\right)$ ) iff it is connecting in $(T, c)$. Since $\alpha \in M_{c}(x) \cap M_{c}(y)$ and $\alpha$ is not used by any connecting edge in $(T, c), \alpha$ is not used by any connecting edge in $\left(T_{r} \cup Y^{\prime}, c\right)$ (respectively, $\left.\left(T_{r-1} \cup Y^{\prime}, c\right)\right)$. So $\{x, y\}$ is a good augmenting pair in $\left(T_{r} \cup Y^{\prime}, c\right)$ (respectively, $\left.T_{r-1} \cup Y^{\prime}\right)$. Now there exists some truncation of $\left(T_{r} \cup Y^{\prime}, c\right)$ (respectively, $\left.\left(T_{r-1} \cup Y^{\prime}, c\right)\right)$ which induces a VKT-tree $\left(T^{\prime}, c\right)$ in $(G, a b, c)$ with top $y^{\prime}$ such that $\left(T^{\prime}-y^{\prime}, c\right)$ is nonaugmenting, but $y^{\prime}$ is contained in an augmenting pair in $\left(T^{\prime}, c\right)$. We may assume $y^{\prime}$ is contained in a good augmenting pair in $\left(T^{\prime}, c\right)$; for otherwise it follows from Lemma 4.1 that (1) or (2) holds. Hence (1) holds with $\left(T^{\prime}, c\right)$.

So we may assume $Y \cap T_{r}=\emptyset$. Then $Y^{\prime}=Y$ and $Y \subseteq G-T_{r}$. Moreover, $y^{*} \notin T$; as otherwise, we have $y^{*} \in(T-y)-T_{r}$ and $\{\alpha, \beta\} \cap M_{c}\left(y^{*}\right) \neq \emptyset$, which implies that $\left\{y^{*}, x\right\}$ or $\left\{y^{*}, z\right\}$ is an augmenting pair in $\left(T_{m-1}, c\right)$, a contradiction. Let $c^{\prime}$ denote the partial $k$-edgecoloring of $G$ obtained from $c$ by interchanging $Y$. Note that $V\left(T_{m-1}\right)$ is nonaugmenting with respect to $c^{\prime}$.

Since $Y \subseteq G-T_{r}$, it follows from Lemma 3.1 that $\left(T_{r}, c^{\prime}\right)$ is a VKT-tree in $\left(G, a b, c^{\prime}\right)$, and any edge of $T_{r}$ is connecting in $\left(T_{r}, c^{\prime}\right)$ iff it is also connecting in $(T, c)$.

We claim that for any $r+1 \leq i \leq m, c^{\prime}\left(e_{i}\right) \in M_{c^{\prime}}\left(T_{i-1}\right)$. If $c\left(e_{i}\right) \notin\{\alpha, \beta\}$, then by Claim $2, c^{\prime}\left(e_{i}\right)=c\left(e_{i}\right) \in M_{c}\left(T_{i-1}\right)=M_{c^{\prime}}\left(T_{i-1}\right)$ (since $\left.y^{*} \notin T\right)$. Now assume $c\left(e_{i}\right) \in\{\alpha, \beta\}$. Then, since $Y \subseteq G-T_{r}, c^{\prime}\left(e_{i}\right) \in\{\alpha, \beta\} \subseteq M_{c^{\prime}}(\{x, z\}) \subseteq M_{c^{\prime}}\left(T_{i-1}\right)$.

Therefore by repeatedly applying Lemma $2.5(a)$, we deduce that $\left(T, c^{\prime}\right)$ is a VKT-tree in $\left(G, a b, c^{\prime}\right)$. Again since $Y \subseteq G-T_{r}$, we must have $\beta \in M_{c^{\prime}}(y) \cap M_{c^{\prime}}(z)$. Moreover, any edge of $T$ is connecting in $\left(T, c^{\prime}\right)$ iff it is connecting in $(T, c)$. Hence, Claim 3 holds with $c^{\prime}, z, \beta$ as $c, x, \alpha$, respectively.

Claim 4. We may assume $m=r+1$. (This may require one interchange.) 
Suppose $m \geq r+2$. Let $z$ be the end of $e_{r+1}$ with $z \notin T_{r}$. Since $k \geq \Delta(G)+\sqrt{\Delta(G) / 2}$ and by Lemma 3.6(2), we may choose $\beta \in M_{c}(z)-c\left(T_{r+2}\right)$. If $\beta \in M_{c}(y)$, then (3) holds, with good augmenting pair $\{y, z\}$ in $(T, c)$ (by Claim 2). So we may assume $\beta \notin M_{c}(y)$.

By Claim 3 and by the choice of $\beta,\{\alpha, \beta\} \cap c\left(T_{r+1}\right)=\emptyset$. So we may apply Lemma 3.4 (with $q:=r+1$ ).

If Lemma 3.4(2) holds then (2) holds. If Lemma 3.4(3) holds then (3) holds since $z \notin$ $C(T, c)$.

So we may assume that Lemma 3.4(1) holds. Then there is a component $A$ of $G_{c}(\alpha, \beta)$ such that $|V(A) \cap\{x, z\}|=1$, and if $c^{\prime}$ denotes the partial $k$-edge-coloring of $G$ obtained from $c$ by interchanging $A$, then $\{\alpha, \beta\} \cap M_{c^{\prime}}(x) \cap M_{c^{\prime}}(z) \neq \emptyset,\left(T_{r+1}, c^{\prime}\right)$ is a VKT-tree in $\left(G, a b, c^{\prime}\right)$, and any edge of $T_{r+1}$ is connecting in $\left(T_{r+1}, c^{\prime}\right)$ iff it is connecting in $(T, c)$. Moreover, $M_{c^{\prime}}(v)=M_{c}(v)$ for all $v \in V\left(T_{r}\right)$ except possibly $M_{c^{\prime}}(x)=\left(M_{c}(x)-\{\alpha\}\right) \cup\{\beta\}$. Therefore, since $\left(T_{m-1}, c\right)$ is nonaugmenting, $\left(T_{r}, c^{\prime}\right)$ is nonaugmenting. Because $\{\alpha, \beta\} \cap c\left(T_{r+1}\right)=\emptyset$, $\{z, x\}$ is a good augmenting pair in $\left(T_{r+1}, c^{\prime}\right)$. So (1) holds, completing the proof of Claim 4.

By Claim 3 and Claim $4, \alpha \notin c(T)$. Let $\beta:=c\left(e_{m}\right)$. Then $\beta \neq \alpha$, since $\alpha \in M_{c}(y)$. We may assume that $e_{m}$ is not a connecting edge in $(T, c)$; as otherwise (2) holds by Lemma 3.3. So $\beta \in M_{c}\left(T_{r}\right)$.

Claim 5. We may assume $\beta \in M_{c}(w)$.

Otherwise, $\beta \in M_{c}\left(T_{m-2}\right)$. Then by Lemma $2.5(a),\left(T_{m-2}+e_{m}, c\right)$ is a VKT-tree in $(G, a b, c)$ with edge-ordering $e_{1}, \ldots, e_{m-2}, e_{m}$. Clearly, $C\left(T_{m-2}+e_{m}, c\right) \subseteq T_{r-1}$, which is properly contained in $C(T, c)=T_{r}$. So $\left(T_{m-2}+e_{m}, c\right)<_{1}(T, c)$. It is also clear that $S\left(T_{m-2}+\right.$ $\left.e_{m}, c\right)$ is a truncation of $S(T, c)$, and any edge of $T_{m-2}+e_{m}$ is connecting in $\left(T_{m-2}+e_{m}, c\right)$ iff it is connecting in $(T, c)$. So $(1)$ holds with $T^{\prime}:=T_{m-2}+e_{m}$ and $c^{\prime}:=c$. Therefore we may assume $\beta \in M_{c}(w)$.

We now distinguish two cases according to whether or not $\beta$ is used by a connecting edge in $(T, c)$. Note that in each case we may need to apply two interchanges.

Case 1. $\beta$ is not used by any connecting edge in $(T, c)$.

Then, since $\left(T_{m-1}, c\right)$ is nonaugmenting, it follows from Lemma 3.6(1) that $\beta \notin c\left(T_{r}\right)$. By Claim 3, $\alpha \notin c\left(T_{r+1}\right)$. Hence we can apply Lemma 3.4 with $q:=r$ and $z:=w$. If Lemma 3.4(2) holds, then (2) holds.

Now suppose Lemma 3.4(1) holds. Then there is a component $A$ of $G_{c}(\alpha, \beta)$ such that $|V(A) \cap\{w, x\}|=1$, and if $c^{\prime}$ denotes the partial $k$-edge-coloring of $G$ obtained from $c$ by interchanging $A$, then $\{\alpha, \beta\} \cap M_{c^{\prime}}(w) \cap M_{c^{\prime}}(x) \neq \emptyset,\left(T_{r}, c^{\prime}\right)$ is a VKT-tree in $\left(G, a b, c^{\prime}\right), S\left(T_{r}, c^{\prime}\right)$ is a truncation of $S(T, c)$, and any edge of $T_{r}$ is connecting in $\left(T_{r}, c^{\prime}\right)$ iff it is connecting in $(T, c)$. Moreover, $M_{c^{\prime}}(v)=M_{c}(v)$ for all $v \in V\left(T_{r-1}\right)$ except possibly $M_{c^{\prime}}(x)=\left(M_{c}(x)-\{\alpha\}\right) \cup\{\beta\}$. Hence, since $\left(T_{m-1}, c\right)$ is nonaugmenting, $\left(T_{r-1}, c^{\prime}\right)$ is nonaugmenting. Since $\alpha, \beta \notin c\left(T_{r}\right)$, $\{w, x\}$ is a good augmenting pair in $\left(T_{r}, c^{\prime}\right)$. So (1) holds with $T^{\prime}:=T_{r}$.

We thus may assume that Lemma 3.4(3) holds. Then there is a component $A$ of $G_{c}(\alpha, \beta)$ intersecting $\{w, x, y\}$ such that $|V(A) \cap\{x, w\}| \neq 1$, and if $c^{*}$ denotes the partial $k$-edgecoloring of $G$ obtained from $c$ by interchanging $A$, then $\{\alpha, \beta\} \cap M_{c^{*}}(w) \cap M_{c^{*}}(y) \neq \emptyset,\left(T, c^{*}\right)$ is a VKT-tree in $\left(G, a b, c^{*}\right)$ with edge ordering $e_{1}, \ldots, e_{m}$, and any edge of $T$ is connecting in $\left(T, c^{*}\right)$ iff it is connecting in $(T, c)$. Since $|V(A) \cap\{x, w\}| \neq 1, c^{*}\left(e_{m}\right) \in M_{c^{*}}\left(T_{m-2}\right)$. Note that $\left(T_{m-1}, c^{*}\right)$ is nonaugmenting. Since $\{\alpha, \beta\} \subseteq M_{c^{*}}\left(T_{r}\right)-c^{*}\left(T_{r}\right)$, no connecting edge in $\left(T, c^{*}\right)$ uses $\alpha$ or $\beta$. So $\{y, w\}$ is a good augmenting pair in $\left(T, c^{*}\right)$. 
We only treat the case when $\alpha \in M_{c^{*}}(y) \cap M_{c^{*}}(w)$; the case when $\beta \in M_{c^{*}}(y) \cap M_{c^{*}}(w)$ can be treated in the same way.

By Lemma 3.5(2) (when $m \geq 4$ ), there exist $z \in V\left(T_{m-2}\right) \cap V_{0}\left(T, c^{*}\right)$ and $\gamma \in M_{c^{*}}(z)-$ $(c(T) \cup\{\alpha\}$ ), which also holds when $m=3$ (since we assume $\Delta(G) \geq 3$ ). Therefore, we may apply Lemma 3.4 (with $q:=m-1$, and with $c^{*}, w, \gamma$ as $c, x, \beta$, respectively). If Lemma 3.4(2) holds then (2) holds.

Now suppose that Lemma 3.4(1) holds. Then there is a component $A$ of $G_{c^{*}}(\alpha, \gamma)$ such that $|V(A) \cap\{w, z\}|=1$, and if $c^{\prime}$ denotes the partial $k$-edge-coloring of $G$ obtained from $c^{*}$ by interchanging $A$, then $\{\alpha, \gamma\} \cap M_{c^{\prime}}(w) \cap M_{c^{\prime}}(z) \neq \emptyset,\left(T_{m-1}, c^{\prime}\right)$ is a VKT-tree in $\left(G, a b, c^{\prime}\right)$, $S\left(T_{m-1}, c^{\prime}\right)$ is a truncation of $S(T, c)$, and any edge of $T_{m-1}$ is connecting in $\left(T_{m-1}, c^{\prime}\right)$ iff it is connecting in $\left(T, c^{*}\right)$. Note that $M_{c^{\prime}}(v)=M_{c^{*}}(v)$ for all $v \in V\left(T_{m-2}-z\right)$, and $M_{c^{\prime}}(z)=M_{c^{*}}(z)$ or $M_{c^{\prime}}(z)=\left(M_{c^{*}}(z)-\{\gamma\}\right) \cup\{\alpha\}$. Then because $\left(T_{m-1}, c^{*}\right)$ is nonaugmenting, $\left(T_{m-2}, c^{\prime}\right)$ is nonaugmenting. Since $\alpha, \gamma \notin c\left(T_{r}\right),\{w, z\}$ is a good augmenting pair in $\left(T_{m-1}, c^{\prime}\right)$. Hence (1) holds.

So assume that Lemma 3.4(3) holds. Then there is a component $A$ of $G_{c^{*}}(\alpha, \gamma)$ intersecting $\{w, y, z\}$ such that $|V(A) \cap\{z, w\}| \neq 1$, and if $c^{\prime}$ denotes the partial $k$-edge-coloring of $G$ obtained from $c^{*}$ by interchanging $A$, then $\{\alpha, \gamma\} \cap M_{c^{*}}(y) \cap M_{c^{*}}(z) \neq \emptyset,\left(T, c^{\prime}\right)$ is a VKT-tree in $\left(G, a b, c^{\prime}\right)$ with edge ordering $e_{1}, \ldots, e_{m}$, and any edge of $T$ is connecting in $\left(T, c^{\prime}\right)$ iff it is connecting in $\left(T, c^{*}\right)$. In particular, $\left(T_{m-2}, c^{\prime}\right)$ is a VKT-tree in $\left(G, a b, c^{\prime}\right)$. However, since $c^{*}\left(e_{m}\right) \in M_{c^{*}}\left(T_{m-2}\right)-\{\alpha, \gamma\}, c^{\prime}\left(e_{m}\right) \in M_{c^{\prime}}\left(T_{m-2}\right)$. By Lemma 2.5 $(a),\left(T_{m-2}+e_{m}, c^{\prime}\right)$ is a VKTtree in $\left(G, a b, c^{\prime}\right)$ with edge ordering $e_{1}, \ldots, e_{m-2}, e_{m}$. Note that $S\left(T_{m-2}+e_{m}, c^{\prime}\right)$ is a truncation of $S(T, c)$. Since $|V(A) \cap\{z, w\}| \neq 1$, we must have $\{z, w\} \subseteq V(A)$ or $V(A) \cap\{w, y, z\}=\{y\}$. So $M_{c^{\prime}}(v)=M_{c^{*}}(v)$ for all $v \in V(T)-\{w, y, z\}$, and either $M_{c^{\prime}}(z)=M_{c^{*}}(z)$ and $M_{c^{\prime}}(w)=$ $M_{c^{*}}(w)$ or $M_{c^{\prime}}(z)=\left(M_{c^{*}}(z)-\{\gamma\}\right) \cup\{\alpha\}$ and $M_{c^{\prime}}(w)=\left(M_{c^{*}}(w)-\{\alpha\}\right) \cup\{\gamma\}$. Then, since $\left(T_{m-1}, c^{*}\right)$ is nonaugmenting, $\left(T_{m-1}, c^{\prime}\right)$ is nonaugmenting. Since $\alpha, \gamma \notin c\left(T_{m-2}+e_{m}\right),\{y, z\}$ is a good augmenting pair in $\left(T_{m-2}+e_{m}, c^{\prime}\right)$. Therefore, since $\left(T_{m-2}+e_{m}, c^{\prime}\right)<_{1}\left(T, c^{*}\right)$, $\left(T_{m-2}+e_{m}, c^{\prime}\right)<_{1}(T, c)$; and (1) holds.

Case 2. $\beta$ is used by some connecting edge in $(T, c)$

In particular, $(T, c)$ has a connecting edge. So let $e_{s}$ denote the last connecting edge in $(T, c)$. Then by Claims 2 and 5 and since $\left(T_{m-1}, c\right)$ is nonaugmenting, $\beta \neq c\left(e_{j}\right)$ for $s+1 \leq j \leq m-1$. Let $A^{\prime}$ denote the component of $G_{c}(\alpha, \beta)$ containing $x$.

We may assume that $A^{\prime}$ contains all edges in $\left[T_{i-1}, G-T_{i-1}\right]$ that use color $\beta$, whenever $e_{i}$ is a connecting edge in $(T, c)$; for, otherwise, since $x \in V_{0}(T, c)$ and $\alpha \notin c(T)$ (by Claim 3), it follows from Lemma 3.2 that (2) holds.

Since $x \in A^{\prime},\{w, y\} \nsubseteq V\left(A^{\prime}\right)$. So there is a component of $G_{c}(\alpha, \beta)$, say $A$, intersecting $\{w, y\}$ such that $A$ is disjoint from $A^{\prime}$. This implies $A \subseteq G-T_{s-1}$, since $A^{\prime}$ uses all edges in $\left[T_{s-1}, G-T_{s-1}\right]$ with color $\beta$ and $\alpha$ is not used by any edge in $\left[T_{s-1}, G-T_{s-1}\right]$.

Let $c^{*}$ denote the partial $k$-edge-coloring of $G$ obtained from $c$ by interchanging $A$. Then by Lemma $3.1,\left(T_{s}, c^{*}\right)$ is a VKT-tree in $\left(G, a b, c^{*}\right)$, and any edge of $T_{s}$ is connecting in $\left(T_{s}, c^{*}\right)$ iff it is connecting in $(T, c)$. Recall that $\alpha \notin c(T), \beta \neq c\left(e_{j}\right)$ for $s+1 \leq j \leq m-1$, and $A \subseteq G-T_{s-1}$. Also note that both ends of $A$ are disjoint from $T_{m-2}$. So $c^{*}\left(e_{j}\right) \in M_{c^{*}}\left(T_{j-1}\right)$ for $s+1 \leq j \leq m-1$. Hence by repeatedly applying Lemma 2.5(a), $\left(T_{m-1}, c^{*}\right)$ is a VKT-tree in $\left(G, a b, c^{*}\right)$.

Suppose $w \notin A^{\prime}$. Then we may choose $A$ so that $w \in A$. So $\left(T_{m-1}, c^{*}\right)$ is a VKT-tree in $\left(G, a b, c^{*}\right),\left(T_{m-2}, c^{*}\right)$ is nonaugmenting, and $\alpha \in M_{c^{*}}(x) \cap M_{c^{*}}(w)$. Since $\alpha \notin c(T), \beta \neq c\left(e_{j}\right)$ 
for $s+1 \leq j \leq m-1$, and $A \subseteq G-T_{s-1}$, we have $\alpha \notin c^{*}\left(T_{m-1}\right)$. So $\{x, w\}$ is a good augmenting pair in $\left(T_{m-1}, c^{*}\right)$. Hence, (1) holds with $T^{\prime}:=T_{m-1}$ and $c^{\prime}:=c^{*}$.

Therefore, we may assume $w \in A^{\prime}$. Then $y \in A, c^{*}\left(e_{m}\right)=\alpha \in M_{c^{*}}\left(T_{m-2}\right)$, and $\beta \neq c^{*}\left(e_{j}\right)$ for $s+1 \leq j \leq m$. Hence by Lemma $2.5(a),\left(T_{m}, c^{*}\right)=\left(T, c^{*}\right)$ is a VKT-tree in $\left(G, a b, c^{*}\right)$. Moreover, $\beta \in M_{c^{*}}(y) \cap M_{c^{*}}(w),\left(T_{m-1}, c^{*}\right)$ is nonaugmenting, and any edge of $T$ is connecting in $\left(T, c^{*}\right)$ iff it is connecting in $(T, c)$.

By Lemma 3.5(2) (and since we assume $\Delta(G) \geq 3$ ), there exist $z \in V_{0}\left(T, c^{*}\right)$ and $\gamma \in$ $M_{c^{*}}(z)-\left(c^{*}(T) \cup\{\alpha\}\right)$. We may assume that the component of $G_{c^{*}}(\gamma, \beta)$ containing $z$, say $B^{\prime}$, contains all edges in $\left[T_{s-1}, G-T_{s-1}\right]$ that use color $\beta$; for, otherwise, since $z \in V_{0}\left(T, c^{*}\right)$, it follows from Lemma 3.2 that (2) holds. Therefore, there is a component of $G_{c^{*}}(\gamma, \beta)$, say $B$, intersecting $\{w, y\}$ such that $B \cap B^{\prime}=\emptyset$. Hence $B \subseteq G-T_{s-1}$. Let $c^{\prime}$ denote the partial $k$-edge-coloring of $G$ obtained from $c^{*}$ by interchanging $B$. Then by Lemma 3.1, $\left(T_{s}, c^{\prime}\right)$ is a VKT-tree in $\left(G, a b, c^{\prime}\right)$, and any edge of $T_{s}$ is connecting in $\left(T_{s}, c^{\prime}\right)$ iff it is connecting in $\left(T, c^{*}\right)$ (iff it is connecting in $(T, c)$ ). Recall that $\gamma \notin c^{*}(T)$ and $\beta \neq c^{*}\left(e_{j}\right)$ for $s+1 \leq j \leq m$, and note that both ends of $B$ are disjoint from $T_{m-2}$. Hence, since $e_{s}$ is the last connecting edge in $\left(T, c^{*}\right)$ and because $B \subseteq G-T_{s-1}, c^{\prime}\left(e_{j}\right) \in M_{c^{\prime}}\left(T_{j-1}\right)$ for $s+1 \leq j \leq m$. So by Lemma $2.5(a)$, $\left(T_{m}, c^{\prime}\right)$ is a VKT-tree in $\left(G, a b, c^{\prime}\right)$. Clearly, any edge of $T$ is connecting in $\left(T, c^{\prime}\right)$ iff it is connecting in $\left(T, c^{*}\right)$ (iff it is connecting in $(T, c)$ ).

Suppose $w \notin B^{\prime}$. Then we may choose $B$ so that $w \in B$. In this case, $\left(T_{m-1}, c^{\prime}\right)$ is a VKT-tree, $\left(T_{m-2}, c^{\prime}\right)$ is nonaugmenting, and $\gamma \in M_{c^{\prime}}(z) \cap M_{c^{\prime}}(w)$. Since $B \subseteq G-T_{s-1}$ and $\beta \neq c^{*}\left(e_{j}\right)$ for $s+1 \leq j \leq m$, we have $\gamma \notin c^{\prime}\left(T_{m-1}\right)$, and so $\{z, w\}$ is a good augmenting pair in $\left(T_{m-1}, c^{\prime}\right)$. Hence, $(1)$ holds with $T^{\prime}:=T_{m-1}$.

So we may assume $w \in B^{\prime}$. Then $y \in B$. So $\left(T_{m-1}, c^{\prime}\right)$ is nonaugmenting, $\gamma \in M_{c^{\prime}}(y) \cap$ $M_{c^{\prime}}(z)$, and $c^{\prime}\left(e_{m}\right)=\alpha \in M_{c^{\prime}}\left(T_{m-2}\right)$. By Lemma 2.5 $(a),\left(T_{m-2}+e_{m}, c^{\prime}\right)$ is a VKT-tree in $\left(G, a b, c^{\prime}\right)$. Since $\gamma \notin c^{*}(T), B \subseteq G-T_{s-1}$, and $\beta \neq c^{*}\left(e_{j}\right)$ for $s+1 \leq j \leq m$, we see that $\gamma$ is not used by any connecting edge in $\left(T, c^{\prime}\right)$. So $\{y, z\}$ is a good augmenting pair in $\left(T_{m-2}+e_{m}, c^{\prime}\right)$, and (1) holds with $T^{\prime}:=T_{m-2}+e_{m}$.

The proof of Lemma 4.4 shows that it takes $O(V \Delta)$ time to find the $\left(T^{\prime}, c^{\prime}\right)$ in Lemma 4.4(1), or the $\left(T^{\prime}, c^{\prime}\right)$ in Lemma 4.4(2), or the $\left(T, c^{\prime}\right)$ in Lemma 4.4(3).

\section{Edge-coloring}

We now apply the recoloring lemmas in the previous section to prove Theorem 1.3. First, we prove that if a VKT-tree is augmenting then we can improve it according to $<_{1}$ or $<_{2}$. For a tree $T$ and two vertices $x, y$ of $T$, we use $T[x, y]$ to denote the unique path in $T$ between $x$ and $y$.

Lemma 5.1 Let $(G, a b, c)$ be a $k$-triple with $k \geq \Delta(G)+\sqrt{\Delta(G) / 2}$, let $(T, c)$ be a VKT-tree in $(G, a b, c)$ with edge ordering $e_{1}, \ldots, e_{m}$, let $y$ be the top of $(T, c)$, and assume that $m \geq 2$, $\left(T_{m-1}, c\right)$ is nonaugmenting, and $y$ is in an augmenting pair in $(T, c)$. Then there is a partial $k$-edge-coloring $c^{\prime}$ of $G$ obtained from $c$ by a sequence of $O(V)$ interchanges such that one of the following holds.

(1) There exists a VKT-tree $\left(T^{\prime}, c^{\prime}\right)$ in $\left(G, a b, c^{\prime}\right)$ with top $y^{\prime}$ such that $\left(T^{\prime}, c^{\prime}\right)<_{1}(T, c)$, $\left(T^{\prime}-y^{\prime}, c^{\prime}\right)$ is nonaugmenting, $y^{\prime}$ is in a good augmenting pair in $\left(T^{\prime}, c^{\prime}\right), S\left(T^{\prime}, c^{\prime}\right)$ is a 
truncation of $S(T, c)$, and any edge of $T^{\prime}$ is connecting in $\left(T^{\prime}, c^{\prime}\right)$ iff it is connecting in $(T, c)$.

(2) There is a VKT-tree $\left(T^{\prime}, c^{\prime}\right)$ in $\left(G, a b, c^{\prime}\right)$ such that $(T, c)<_{2}\left(T^{\prime}, c^{\prime}\right)$.

Proof. Let $x \in V\left(T_{m-1}\right)$ such that $\{y, x\}$ is an augmenting pair in $(T, c)$. If $\{x, y\}$ is a good augmenting pair and $x \notin C(T, c)$, apply Lemma 4.3 to $(T, c)$; and if $\{x, y\}$ is a good augmenting pair and $x \in C(T, c)$, apply Lemma 4.4 to $(T, c)$. If $\{x, y\}$ is not a good augmenting pair, apply Lemma 4.1 to $(T, c)$, and then apply Lemma 4.3 or Lemma 4.4 to the resulting VKT-tree.

Then there is a partial $k$-edge-coloring $c^{1}$ of $G$ obtained from $c$ by a sequence of (at most seven) interchanges such that one of the following holds.

(a) There exists a VKT-tree $\left(T^{1}, c^{1}\right)$ in $\left(G, a b, c^{1}\right)$ with top $y^{1}$ such that $\left(T^{1}, c^{1}\right)<_{1}(T, c)$, $\left(T^{1}-y^{1}, c^{1}\right)$ is nonaugmenting, $y^{1}$ is in a good augmenting pair in $\left(T^{1}, c^{1}\right), S\left(T^{1}, c^{1}\right)$ is a truncation of $S(T, c)$, and any edge of $T^{1}$ is connecting in $\left(T^{1}, c^{1}\right)$ iff it is connecting in $(T, c)$.

(b) There is a VKT-tree $\left(T^{1}, c^{1}\right)$ in $\left(G, a b, c^{1}\right)$ such that $(T, c)<_{2}\left(T^{1}, c^{1}\right)$.

(c) $\left(T, c^{1}\right)$ is a VKT-tree in $\left(G, a b, c^{1}\right)$ with edge ordering $e_{1}, \ldots, e_{m},\left(T_{m-1}, c^{1}\right)$ is nonaugmenting, $y$ and a vertex $x_{1}$ of $B\left(T, c^{1}\right)-C\left(T, c^{1}\right)$ form a good augmenting pair in $\left(T, c^{1}\right)$, and any edge of $T$ is connecting in $\left(T, c^{1}\right)$ iff it is connecting in $(T, c)$.

If (a) or (b) holds, we see that (1) or (2) holds, with $T^{\prime}:=T^{1}$ and $c^{\prime}:=c^{1}$. So we may assume that (c) holds. By repeatedly applying Lemma 4.3 (starting with $\left(T^{1}, c^{1}\right)$ and $x_{1}$ ), we obtain a maximal sequence $\left(\left(T, c^{t}\right), x_{t}: 1 \leq t \leq n\right)$ such that for each $1 \leq t \leq n$, $c^{t}$ is a partial $k$-edge-coloring of $G$ obtained from $c^{t-1}$ by an interchange, $\left(T, c^{t}\right)$ is a VKT-tree in $\left(G, a b, c^{t}\right)$ with edge ordering $e_{1}, \ldots, e_{m},\left(T_{m-1}, c^{t}\right)$ is nonaugmenting, $y$ and a vertex $x_{t}$ of $B\left(T, c^{t}\right)-C\left(T, c^{t}\right)$ form a good augmenting pair in $\left(T, c^{t}\right)$, any edge of $T$ is connecting in $\left(T, c^{t}\right)$ iff it is connecting in $(T, c)$, and $T\left[x_{t}, y\right]$ is a proper subpath of $T\left[x_{t-1}, y\right]$ (for $2 \leq t \leq n$ ).

Note that $\left\{x_{n}, y\right\} \subseteq B\left(T, c^{n}\right)-C\left(T, c^{n}\right)$. By the maximality of the sequence $\left(\left(T^{t}, c^{t}\right), x_{t}\right.$ : $1 \leq t \leq n$ ), it follows from Lemma 4.3 (with $\left(T, c^{n}\right), x_{n}$ as $(T, c), x$, respectively) that there exists a partial $k$-edge-coloring $c^{\prime}$ of $G$ obtained from $c^{n}$ by an interchange such that one of the following holds.

$\left(\mathrm{a}^{\prime}\right)$ There exists a VKT-tree $\left(T^{\prime}, c^{\prime}\right)$ in $\left(G, a b, c^{\prime}\right)$ with top $y^{\prime}$ such that $\left(T^{\prime}, c^{\prime}\right)<_{1}\left(T, c^{n}\right)$, $\left(T^{\prime}-y^{\prime}, c^{\prime}\right)$ is nonaugmenting, $y^{\prime}$ is contained in a good augmenting pair in $\left(T^{\prime}, c^{\prime}\right)$, $S\left(T^{\prime}, c^{\prime}\right)$ is a truncation of $S\left(T, c^{n}\right)$, and any edge of $T^{\prime}$ is connecting in $\left(T^{\prime}, c^{\prime}\right)$ iff it is connecting in $\left(T, c^{n}\right)$,

$\left(\mathrm{b}^{\prime}\right)$ There is a VKT-tree $\left(T^{\prime}, c^{\prime}\right)$ in $\left(G, a b, c^{\prime}\right)$ such that $\left(T, c^{n}\right)<_{2}\left(T^{\prime}, c^{\prime}\right)$.

Note that $S\left(T^{\prime}, c^{\prime}\right)$ is a truncation of $S\left(T, c^{n}\right)=\ldots=S\left(T, c^{1}\right)$, and $C\left(T, c^{n}\right)=\ldots=$ $C\left(T, c^{1}\right)=C(T, c)$. Hence, $\left(T, c^{n}\right)<_{2}\left(T^{\prime}, c^{\prime}\right)$ implies $(T, c)<_{2}\left(T^{\prime}, c^{\prime}\right)$, and $\left(T^{\prime}, c^{\prime}\right)<_{1}\left(T, c^{n}\right)$ implies $\left(T^{\prime}, c^{\prime}\right)<_{1}(T, c)$. So we see that $\left(\mathrm{a}^{\prime}\right)$ or $\left(\mathrm{b}^{\prime}\right)$ implies (1) or (2), respectively.

In the proof of Lemma 5.1, Lemma 4.3 is applied $O(V)$ times. So it takes $O\left(V^{2} \Delta\right)$ time to find the $\left(T^{\prime}, c^{\prime}\right)$ in Lemma 5.1(1), or the $\left(T^{\prime}, c^{\prime}\right)$ in Lemma 5.1(2).

We now prove that given any augmenting VKT-tree, we can either produce a better partial edge-coloring or augment the tree in terms of $<_{2}$. 
Lemma 5.2 Let $(G, a b, c)$ be a k-triple with $k \geq \Delta(G)+\sqrt{\Delta(G) / 2}$, let $(T, c)$ be a VKT-tree in $(G, a b, c)$ with edge ordering $e_{1}, \ldots, e_{m}$, let $y$ be the top of $(T, c)$, and assume that $m \geq 2$, $\left(T_{m-1}, c\right)$ is nonaugmenting, and $y$ is in an augmenting pair in $(T, c)$. Then there is a partial $k$-edge-coloring $c^{\prime}$ of $G$ obtained from $c$ by a sequence of $O\left(V^{2}\right)$ interchanges such that one of the following holds.

(1) $M_{c^{\prime}}(a) \cap M_{c^{\prime}}(b) \neq \emptyset$.

(2) There is a VKT-tree $\left(T^{\prime}, c^{\prime}\right)$ in $\left(G, a b, c^{\prime}\right)$ such that $(T, c)<_{2}\left(T^{\prime}, c^{\prime}\right)$.

Proof. By Lemma 5.1, there is a partial $k$-edge-coloring $c^{\prime}$ of $G$ obtained from $c$ by a sequence of $O(V)$ interchanges such that one of the following holds.

(a) There exists a VKT-tree $\left(T^{1}, c^{1}\right)$ in $\left(G, a b, c^{1}\right)$ with top $y_{1}$ such that $\left(T^{1}, c^{1}\right)<_{1}(T, c)$, $\left(T^{1}-y_{1}, c^{1}\right)$ is nonaugmenting, $y_{1}$ is contained in a good augmenting pair in $\left(T^{1}, c^{1}\right)$, $S\left(T^{1}, c^{1}\right)$ is a truncation of $S(T, c)$, and any edge of $T^{1}$ is connecting in $\left(T^{1}, c^{1}\right)$ iff it is connecting in $(T, c)$.

(b) There is a VKT-tree $\left(T^{1}, c^{1}\right)$ in $G$ such that $(T, c)<_{2}\left(T^{1}, c^{1}\right)$.

If (b) holds, then (2) holds with $T^{\prime}:=T^{1}$ and $c^{\prime}:=c^{1}$. Therefore, we may assume that (a) holds. By repeatedly applying Lemma 5.1 (at most $O(V)$ times and starting with $\left(T^{1}, c^{1}\right)$ and $\left.y_{1}\right)$, we obtain a maximal sequence $\left(\left(T^{t}, c^{t}\right), y_{t}: 1 \leq t \leq n\right)$ such that for each $1 \leq t \leq n, c^{t}$ is a partial $k$-edge-coloring of $G$ obtained from $c^{t-1}$ by a sequence of $O(V)$ interchanges (where $\left.c^{0}=c\right),\left(T^{t}, c^{t}\right)$ is a VKT-tree in $\left(G, a b, c^{t}\right)$ with top $y_{t},\left(T^{t}-y_{t}, c^{t}\right)$ is nonaugmenting, $y_{t}$ is contained in a good augmenting pair in $\left(T^{t}, c^{t}\right),\left(T^{t}, c^{t}\right)<_{1}\left(T^{t-1}, c^{t-1}\right)$ where $\left(T^{0}, c^{0}\right)=(T, c)$, $S\left(T^{t}, c^{t}\right)$ is a truncation of $S\left(T^{t-1}, c^{t-1}\right)$, and any edge of $T^{t}$ is connecting in $\left(T^{t}, c^{t}\right)$ iff it is connecting in $\left(T^{t-1}, c^{t-1}\right)$.

If $V\left(T^{n}\right)=\{a, b\}$, then $M_{c^{n}}(a) \cap M_{c^{n}}(b) \neq \emptyset$; and (1) holds with $c^{\prime}:=c^{n}$.

So we may assume $\left|V\left(T^{n}\right)\right| \geq 3$. Then by the maximality of the sequence $\left(\left(T^{t}, c^{t}\right), y_{t}\right.$ : $1 \leq t \leq n)$ and by Lemma 5.1 , there exists a partial $k$-edge-coloring $c^{\prime}$ of $G$ obtained from $c^{n}$ by a sequence of $O(V)$ interchanges and there is a VKT-tree $\left(T^{\prime}, c^{\prime}\right)$ in $\left(G, a b, c^{\prime}\right)$ such that $\left(T^{n}, c^{n}\right)<_{2}\left(T^{\prime}, c^{\prime}\right)$. Hence $(2)$ holds.

In the proof of Lemma 5.2, Lemma 5.1 is applied $O(V)$ times. So it takes $O\left(V^{3} \Delta\right)$ time to find the $c^{\prime}$ in Lemma 5.2(1) or the $\left(T^{\prime}, c^{\prime}\right)$ in Lemma 5.2(2).

So far, we have shown how to deal with an augmenting VKT-tree. In order to prove Theorem 1.3, we need to deal with nonaugmenting VKT-trees. For this, we introduce another partial ordering on VKT-trees, which refines $<_{2}$.

Definition 5.3 Let $(G, a b, c)$ and $\left(G, a b, c^{\prime}\right)$ be two triples such that $E(c)=E\left(c^{\prime}\right)$, and $c$ and $c^{\prime}$ use the same set of colors. Let $(T, c)$ and $\left(T^{\prime}, c^{\prime}\right)$ be VKT-trees in $(G, a b, c)$ and $\left(G, a b, c^{\prime}\right)$, respectively. We write $(T, c)<_{3}\left(T^{\prime}, c^{\prime}\right)$ if there exists an integer $p \geq 0$ such that

- $S_{j}(T, c)=S_{j}\left(T^{\prime}, c^{\prime}\right)$ for $0 \leq j \leq p-1$, and

- $S_{p}(T, c)$ is a proper truncation of $S\left(T^{\prime}, c^{\prime}\right)$. 
Note the difference between $<_{3}$ and $<_{2}$; here $S_{p}(T, c)$ may be the last segment of $(T, c)$. Also note that any edge of $\bigcup_{j=0}^{p-1} S_{j}\left(T^{\prime}, c^{\prime}\right)$ is connecting in $\left(T^{\prime}, c^{\prime}\right)$ iff it is connecting in $(T, c)$. Clearly, $(T, c)<_{2}\left(T^{\prime}, c^{\prime}\right)$ implies $(T, c)<_{3}\left(T^{\prime}, c^{\prime}\right)$; and $<_{3}$ induces a partial order on the VKT-trees.

The next lemmas says that, given a nonaugmenting VKT-tree, we can either augment it (through $<_{3}$ ) or decide that an additional color is needed.

Lemma 5.4 Let $(G, a b, c)$ be a k-triple with $k \geq \Delta(G)+\sqrt{\Delta(G) / 2}$, and let $(T, c)$ be a nonaugmenting VKT-tree in $(G, a b, c)$. Then one of the following holds.

(1) There is a VKT-tree $\left(T^{\prime}, c\right)$ in $(G, a b, c)$ with top $y^{\prime}$ such that $(T, c)<_{3}\left(T^{\prime}, c\right),\left(T^{\prime}-y^{\prime}, c\right)$ is nonaugmenting, and $y^{\prime}$ is contained in an augmenting pair.

(2) $k<\Gamma(G)$.

Proof. If $(T, c)$ is critical, then (2) follows from Lemma 2.9. So we may assume that $(T, c)$ is not critical.

If $(T, c)$ is not complete, then let $e \in[T, G-T]$ such that $c(e) \in M_{c}(T)$. By Lemma 2.5(a), $(T+e, c)$ is a VKT-tree in $(G, a b, c)$, with edge ordering as in $(T, c)$ followed by $e$. Clearly, $(T, c)<_{3}(T+e, c)$; and let $T^{1}:=T+e$. If $(T, c)$ is complete, then let $\beta$ denote a color that is used by at least two edges in $[T, G-T]$. By Lemma 3.5(2) and since we may assume $\Delta(G) \geq 3$, there exist $x \in V_{0}(T, c)$ and $\alpha \in M_{c}(x)-(c(T) \cup\{\beta\})$. Let $P$ denote the path in $G[T] \cap G_{c}(\alpha, \beta)$ containing $x$, and let $e$ denote the edge in $[T, G-T]$ with color $\beta$ and incident with $P$. Now by Lemma $2.5(b),(T+e, c)$ is a VKT-tree in $(G, a b, c)$, with edge ordering as in $(T, c)$ followed by $e$; and let $T^{1}:=T+e$.

If $\left(T^{1}, c\right)$ is augmenting, then (1) holds; otherwise, we apply the above argument to $\left(T^{1}, c^{1}\right)$ (in place of $(T, c))$. Now let $\left(\left(T^{t}, c\right): 1 \leq t \leq n\right)$ denote the maximal sequence of VKT-trees (with top $y_{t}$ ) constructed recursively using the above process, such that $\left(T^{t}, c\right)<_{3}\left(T^{t+1}, c\right)$ for $1 \leq t \leq n-1$, and $\left(T^{n}-y_{n}, c\right)$ is nonaugmenting. If $\left(T^{n}, c\right)$ is augmenting, then (1) holds. If $\left(T^{n}, c\right)$ is nonaugmenting, then by the maximality of this sequence, $\left(T^{n}, c\right)$ is critical. Hence by Lemma $2.9,(2)$ holds.

It is easy to see that it takes $O(E)$ time to find the $\left(T^{\prime}, c^{\prime}\right)$ in Lemma 5.4(1) or to certify that Lemma 5.4(2) holds.

We now prove that given a $k$-triple $(G, a b, c)$ with $k \geq \Delta(G)+\sqrt{\Delta(G) / 2}$, we can modify $c$ to a "better" partial edge-coloring or show that a new color must be introduced.

Lemma 5.5 Let $(G, a b, c)$ be a $k$-triple with $k \geq \Delta(G)+\sqrt{\Delta(G) / 2}$. Then one of the following holds.

(1) There is a partial edge-coloring $c^{\prime}$ obtained from $c$ by a sequence of $O\left(V^{3}\right)$ interchanges such that $M_{c^{\prime}}(a) \cap M_{c^{\prime}}(b) \neq \emptyset$.

(2) $k<\Gamma(G)$.

Proof. Start with the VKT-tree $\left(T^{1}, c^{1}\right)$ induced by the edge $a b$, with $c^{1}=c$. If $M_{c^{1}}(a) \cap$ $M_{c^{1}}(b) \neq \emptyset$, then $(1)$ holds with $c^{\prime}:=c^{1}$. So we may assume $\left(T^{1}, c^{1}\right)$ is nonaugmenting. By Lemma 5.4, either (2) holds, or there is a VKT-tree $\left(T^{2}, c^{2}\right)$ in $\left(G, a b, c^{2}\right)$ with top $y_{2}$ (and with 
$\left.c^{2}=c^{1}\right)$ such that $\left(T^{1}, c^{1}\right)<_{3}\left(T^{2}, c^{2}\right),\left(T^{2}-y_{2}, c^{2}\right)$ is nonaugmenting, and $y_{2}$ is contained in an augmenting pair.

By Lemma 5.2, either (1) holds, or there is a partial edge-coloring $c^{3}$ obtained from $c^{2}$ by a sequence of $O\left(V^{2}\right)$ interchanges and there exists a VKT-tree $\left(T^{3}, c^{3}\right)$ in $\left(G, a b, c^{3}\right)$ such that $\left(T^{2}, c^{2}\right)<_{2}\left(T^{3}, c^{3}\right)$. Hence $\left(T^{2}, c^{2}\right)<_{3}\left(T^{3}, c^{3}\right)$.

So let $\left(\left(T^{t}, c^{t}\right): 1 \leq t \leq n\right)$ denote the maximal sequence of VKT-trees obtained as above, such that $\left(T^{t}, c^{t}\right)<_{3}\left(T^{t+1}, c^{t+1}\right)$ for $1 \leq t \leq n-1$.

If $\left(T^{n}, c^{n}\right)$ is nonaugmenting, then we apply Lemma 5.4 and see that (2) holds (by the maximality of the sequence). If $\left(T^{n}, c^{n}\right)$ is augmenting, then we may apply Lemma 5.2 and see that (1) holds.

By Lemma 3.5(1), each VKT-tree contains at most $\lceil\sqrt{\Delta}\rceil$ segments. So the $n$ in the sequence $\left(\left(T^{t}, c^{t}\right): 1 \leq t \leq n\right)$ could be as large as $O\left(V^{O(\sqrt{\Delta})}\right)$. Note that $E=O(V \Delta)$. Hence, it takes $O\left(V^{O(\sqrt{\Delta})+3} \Delta\right)$ time to find the $c^{\prime}$ in Lemma 5.5(1) or certify Lemma 5.5(2).

Proof of Theorem 1.3. Let $G$ be a multigraph. We may assume $\Delta(G) \geq 3$ since otherwise the assertion of Theorem 1.3 holds.

First, greedily color as many edges of $G$ as possible using $\Delta(G)$ colors. Let $c$ denote the resulting partial edge-coloring of $G$.

If $E(c)=E(G)$, then $c$ gives the desired edge-coloring. So we may assume $E(c) \neq E(G)$. Choose an arbitrary edge $a b$ from $E(G)-E(c)$, and apply Lemma 5.5 to the triple $(G, a b, c)$.

If Lemma 5.5(1) holds, we obtain a partial edge-coloring $c^{\prime}$ of $G$ from $c$ through a sequence of interchanges such that $M_{c^{\prime}}(a) \cap M_{c^{\prime}}(b) \neq \emptyset$. Let $c_{1}$ denote the partial edge-coloring of $G$ obtained from $c$ by coloring $a b$ with a color from $M_{c^{\prime}}(a) \cap M_{c^{\prime}}(b)$. If Lemma 5.5(2) holds, then $k<\Gamma(G)$, and let $c_{1}$ denote the partial edge-coloring of $G$ obtained from $c$ by assigning to the edge $a b$ a color not used by $c$.

Repeat this argument for $G$ and $c_{1}$, we obtain the desired edge-coloring of $G$. (This takes at most $O(E)$ steps.)

Clearly, the above argument gives an algorithm for edge-coloring any multigraph $G$ using at most $\max \{\Delta(G)+\sqrt{\Delta(G) / 2},\lceil\Gamma(G)\rceil\}$ colors. The complexity of this algorithm is $O\left(V^{O(\sqrt{\Delta})+3} \Delta E\right)$. So if $\Delta$ is not part of the input, our proof yields a polynomial time coloring algorithm.

Proof of Corollary 1.5. Let $G$ be a multigraph, and let $c$ be any given edge-coloring of $G$, using colors from the collection $\mathcal{C}$. Let $k:=|\mathcal{C}|$.

Pick a color $\alpha \in \mathcal{C}$, and let $E_{c}(\alpha):=\{e \in E(G): c(e)=\alpha\}$. Let $c_{1}$ denote the partial edgecoloring of $G$ with $E\left(c_{1}\right)=E(G)-E(\alpha)$, and $c_{1}(e)=c(e)$ for each $e \in E(G)-E(\alpha)$. (This is for notational purpose only; we may simply view $c_{1}$ as $c$ with $\alpha$ ignored.) Our objective is to show that either $k=\chi^{\prime}(G)$ (in which case, $c$ is an optimal edge-coloring) or use a sequence of interchanges to turn $c$ to an edge-coloring of $G$ using colors from $\mathcal{C}-\{\alpha\}$; and by repeating this process we will produce an optimal edge-coloring of $G$.

Pick an edge $a b$ from $E(\alpha)$. Apply Lemma 5.5 to the $(k-1)$-triple $\left(G, a b, c_{1}\right)$.

If Lemma 5.5(2) holds, then $k-1<\Gamma(G)$; thus $k=\lceil\Gamma(G)\rceil$, and so, $c$ is an optimal edge-coloring of $G$.

So we may assume that Lemma 5.5(1) holds. Then there is a partial edge-coloring $c_{1}^{\prime}$ obtained from $c_{1}$ by a sequence of interchanges such that $M_{c_{1}^{\prime}}(a) \cap M_{c_{1}^{\prime}}(b) \neq \emptyset$. Let $\beta \in$ 
$M_{c_{1}^{\prime}}(a) \cap M_{c_{1}^{\prime}}(b)$. Note that $\beta \in \mathcal{C}-\{\alpha\}$. Let $c_{1}^{*}$ denote the edge-coloring of $G$ obtained from $c_{1}^{\prime}$ by coloring the edges in $E(\alpha)$ with color $\alpha$ again. Clearly, $c_{1}^{*}$ can be obtained from $c$ by a sequence of interchanges. Let $D$ denote the component of $G_{c_{1}^{*}}(\alpha, \beta)$ containing the edge $a b$. Then $D$ is induced by the edge $a b$. Let $c_{2}$ denote the edge-coloring of $G$ obtained from $c_{1}^{*}$ by interchanging $D$. Then $E_{c_{2}}(\alpha)=E_{c}(\alpha)-\{a b\}$.

We repeat the above argument for $c_{2}$ by picking an edge from $E_{c_{2}}(\alpha)$. We either show that $c_{2}$ is an optimal edge-coloring, or obtain an edge-coloring of $G$ using colors from $\mathcal{C}-\{\alpha\}$. If the latter occurs, we pick a color from $\mathcal{C}-\{\alpha\}$ and repeat the whole process again. (We omit the details.) Eventually, we obtain an optimal edge-coloring of $G$.

\section{References}

[1] L.D. Andersen, On edge-colorings of graphs, Math. Scand. 40 (1977), 161-175.

[2] A. Caprara and R. Rizzi, Improving a family of approximation algorithms to edge color multigraphs, Information Processing Letters 68 (1998) 11-15.

[3] J. Edmonds, Maximum matching and a polyhedron with 0,1-vertices, J. Res. Nat. Bureau Standards (B) 69 (1965) 125-130.

[4] L.M. Favrholdt, M. Stiebitz and B. Toft, Graph edge colouring: Vizing's theorem and Goldberg's conjecture, Preprint (bib.mathematics.dk).

[5] M.K. Goldberg, On multigraphs of almost maximal chromatic class (Russian), Discret. Analiz. 23 (1973), 3-7.

[6] M.K. Goldberg, Edge-coloring of multigraphs: recoloring technique, J. Graph Theory 8 (1984), 123-137.

[7] R. P. Gupta, Studies in the theory of graphs Ph.D thesis, Tata Institute of Fundamental Research, Bombay, 1967.

[8] D.S. Hochbaum, T. Nishizeki and D.B. Shmoys, A better than "best possible" algorithm to edge color multigraphs Journal of Algorithms 7 (1986) 79-104.

[9] I.J. Holyer, The NP-completeness of edge-colorings, SIAM J. Comput. 10 (1980), 718-720.

[10] T.R. Jensen and B. Toft, Graph Coloring Problems, Wiley-Intersci. Ser. Discrete Math. Optim., Wiley, New York 1994.

[11] J. Kahn, Asymptotics of the chromatic index for multigraphs, J. Combin. Theory Ser. B 68 (1996), 233-254.

[12] H.A. Kierstead, On the chromatic index of multigraphs without large triangles, J. Combin. Theory Ser. B 36 (1984) 156-160.

[13] O. Marcotte, On the chromatic index of multigraphs and a conjecture of Seymour, I, $J$. Combin. Theory Ser. B 41 (1986) 306-331.

[14] O. Marcotte, Exact edge-colorings of graphs without prescribed minors, in: Polyhedral Combinatorics (W. Cook and P. Seymour, eds.), DIMACS Series in Discrete Mathematics and Theoretical Computer Science 1 (1990) 235-243.

[15] O. Marcotte, On the chromatic index of multigraphs and a conjecture of Seymour, II, in: Polyhedral Combinatorics (W. Cook and P. Seymour, eds.), DIMACS Series in Discrete Mathematics and Theoretical Computer Science 1 (1990) 245-279. 
[16] T. Nishizeki and K. Kashiwagi, On the 1.1 edge-coloring of multigraphs, SIAM J. Discrete Math. 3 (1990) 391-410.

[17] M.J. Plantholt and S.K. Tipnis, Regular multigraphs of high degree are 1-factorable, J. London Math. Soc. 44 (1991) 393-400.

[18] M.J. Plantholt, A sublinear bound on the chromatic index of multigraphs, Discrete Math. 202 (1999) 201-213.

[19] P. Sanders and D. Steurer, An asymptotic approximation scheme for multigraph edge coloring, in: Proc. 16th Annual ACM-SIAM Symposium on Discrete Algorithms, (2005) pp. 897-906.

[20] D. Scheide, On the 15/14 edge-colouring of multigraphs, Preprint (bib.mathematics.dk).

[21] A. Schrijver, Combinatorial Optimization - Polyhedra and efficiency, Volume A, SpringerVerlag, Berlin, Heidelberg, New York, 2003.

[22] C. Shannon, A theorem on coloring lines of a network, J. Math. Phys. 28 (1949) 148-151.

[23] P.D. Seymour, On multi-colorings of cubic graphs, and conjectures of Fulkerson and Tutte, Proc. London Math. Soc. 38 (1979) 423-460.

[24] P.D. Seymour, Colouring series-parallel graphs, Combinatorica 4 (1990) 379-392.

[25] V.A. Tashkinov, On an algorithm for the edge coloring of multigraphs (Russian), Diskretn. Anal. Issled. Oper. Ser. 17 (2000), 72-85.

[26] V.G. Vizing, On an estimate of the chromatic class of a p-graph (Russian), Discret. Analiz. 3 (1964), 25-30. 